\title{
RDUS
}

Revue de DROIT UNIVERSITÉ DE SHERBROOKE

Titre : $\quad$ L'OTAN : VERS UNE CONCEPTION ÉLARGIE DE LA SÉCURITÉ

Auteur(s): $\quad$ Roseline MARILLER-RIBET

SUzanne LALONDE

Revue : $\quad$ RDUS, 2006-2007, volume 37, numéro 2

Pages: $\quad 331-391$

ISSN : $\quad 0317-9656$

Éditeur : $\quad$ Université de Sherbrooke. Faculté de droit.

URI : $\quad$ http://hdl.handle.net/11143/11838

DOI : $\quad$ https://doi.org/10.17118/11143/11838 
Page vide laissée intentionnellement. 


\title{
ARTICLE
}

\section{L'OTAN : VERS UNE CONCEPTION ÉLARGIE DE LA SÉCURITÉ}

\author{
par Roseline MARILLER-RIBET ${ }^{*}$ \\ Suzanne LALONDE**
}

Les contributions des forces alliées aux opérations militaires menées dans les Balkans, en Méditerranée, en Afghanistan et en Irak témoignent des responsabilités mondiales de plus en plus lourdes qui incombent à l'OTAN. Pour les assumer, l'Alliance s'est lancée dans un processus de transformation et de renouvellement. Cette évolution s'est clairement dessinée au sommet de Washington de 1999 et s'est poursuivie au sommet de Prague de novembre 2002. La refondation complète des structures de l'OTAN représente une nouvelle vision pour l'Organisation et un bouleversement par rapport à l'objectif essentiel original de l'Alliance. La création de cette nouvelle OTAN s'est faite par étapes successives. C'est en grande partie grâce à la mise en place de la Force de réaction de l'OTAN (NRF) et d'une nouvelle structure de commandement que l'OTAN est maintenant en mesure de gérer le nouvel environnement de sécurité et de mener de nouveaux types de missions. L'OTAN a su ainsi s'adapter à ce nouveau contexte stratégique, notamment en acceptant des engagements, d'une part, envers de nouveaux objectifs comme la lutte contre le terrorisme et d'autre part, dans de nouveaux théâtres géographiques comme en Afghanistan, en Irak et au Darfour malgré une transformation en cours de réalisation.

\footnotetext{
* Docteur en droit.

** . Professeure à la Faculté de droit de l'Université de Montréal.
} 
The contributions of the allied military forces carrying out operations in the Balkans, in the Mediterranean, in Afghanistan and in Iraq are clear illustrations of the increasingly onerous tasks assumed by NATO in various regions of the world. In order to properly fulfill these duties, the Alliance has undertaken a process of transformation and renewal. This trend, initiated at the Washington Summit of 1999, was continued at the Prague Summit of November 2002. The complete reorganization of NATO's structures represents not only a new vision for the Alliance but also a disruption of sorts in light of the original fundamental objective of the Alliance. The setting up of this "new" NATO has been incremental and it is mainly due to the creation of the NATO Response Force and to the introduction of a revised command structure that the Alliance is now able to react to the new security environment and to pursue new types of missions. Consequently, despite the transformation it is presently undergoing, NATO has been able to adapt to the changing strategic context by accepting missions such as the fight against terrorism and by entering new theatres of operation such as Afghanistan, Iraq and Darfour 
L'OTAN :

(2007) 37 R.D.U.S vers une conception élargie

\section{SOMMAIRE}

I. La mise en place de la NRF.

II. La nouvelle structure de commandement

militaire 364

III. Les opérations de l'OTAN 368 

Le Traité de l'Atlantique Nord, qui constitue le fondement juridique et contractuel de l'Alliance atlantique, a été signé le 4 avril 1949 à Washington par la Belgique, le Canada, le Danemark, les États-Unis, la France, l'Islande, l'Italie, le Luxembourg, la Norvège, les Pays-Bas, le Portugal et le Royaume-Uni ${ }^{1}$. Selon son préambule, l'OTAN, qui est la structure qui permet la réalisation des objectifs de l'Alliance, a été créée pour promouvoir des relations pacifiques et amicales dans toute la zone de l'Atlantique Nord $^{2}$. Il s'agissait en fait de prévenir une guerre contre l'URSS sur le théâtre européen et, si nécessaire, de défendre les membres de l'Alliance contre cette menace $^{3}$. Cette mission a été pleinement accomplie puisque, non seulement, il n'y a pas eu de guerre mais encore, l'Union soviétique a disparu sans conflit armé.

Avec la chute du mur de Berlin et l'effondrement de l'URSS, la disparition de la menace pour laquelle l'OTAN avait été mise en place a laissé croire que l'Organisation n'avait plus de raison d'être ${ }^{4}$. L'OTAN aurait donc dû disparaître ${ }^{5}$. Or, l'Organisation a

1. La Grèce et la Turquie ont signé le Traité le 18 février 1952 et jouent un rôle majeur dans le maintien de la sécurité dans la région euro-atlantique et, en particulier sur le flanc sud de l'OTAN. L'Allemagne a adhéré à l'Organisation le 6 mai 1955. L’Espagne a ratifié le Traité le 30 mai 1982 et a adhéré à la structure militaire intégrée en 1998. La République tchèque, la Hongrie et la Pologne ont rejoint l'OTAN le 12 mars 1999. Le 29 mars 2004, la Bulgarie, l'Estonie, la Lettonie, la Lituanie, la Roumanie, la Slovaquie et la Slovénie sont officiellement devenus membres de l'Organisation du Traité de l'Atlantique Nord.

2. OTAN, Manuel de l'OTAN, 3e éd., Bureau de l'information et de la presse de l'OTAN, Bruxelles, 2005 p. 23 [OTAN, "Manuel de l'OTAN"].

3. Ibid.

4. Michael E. Brown, "NATO at Fifty: Minimalist NATO: A Wise Alliance Knows When to Retrench" (1999) 78:3 Foreign Affairs 204 à la p. 204; Frédéric Bozo, "De la bataille des euromissiles à la guerre du Kosovo: l'Alliance atlantique face à ses défis (1979-1999)" (1999) 64 Politique étrangère 587 à la p. 592; Commission politique, "Les nouveaux défis pour la coopération transatlantique en matière de sécurité" (11 mars 2007), en ligne : assemblé interparlementaire européenne de sécurité et de défense <http://www.assembly-weu.org>.

5. Le Pacte de Varsovie, qui était son corollaire, avait disparu tout comme d'autres traités qui avaient été conclus après la Seconde Guerre mondiale. Il en avait été ainsi de l'Otase (Organisation du Traité de l'Asie du Sud-est, 1945-1977) qui regroupait dans une alliance militaire les 
survécu et cela peut s'expliquer par plusieurs raisons. Tout d'abord, les organisations internationales ont tendance à vouloir préserver leur existence ${ }^{6}$. Ensuite, la disparition de l'Union soviétique n'a pas fait pour autant disparaître la menace russe ${ }^{7}$ et des risques de désordre persistent ${ }^{8}$. Enfin, une autre raison réside dans l'incapacité de l'Europe à s'assumer, sur les plans militaire et géostratégique, depuis la fin de la Seconde Guerre mondiale. L'Europe, du fait de son insuffisance à donner une dimension crédible à ses capacités militaires, a créé un vide géopolitique laissant une place pour l'OTAN

D'autres causes circonstancielles, telles que les attentats du 11 septembre 2001, l'influence des néo-conservateurs à Washington $^{9}$ ou les politiques étrangères des différents membres de l'Alliance ${ }^{10}$, ont permis à l'OTAN de se maintenir. L'annonce de

États-Unis, l'Australie, la Nouvelle-Zélande, la Thaïlande, les Philippines, la France et la Grande-Bretagne.

6. Patrick Daillier, Alain Pellet et Nguyen Quoc Dinh, Droit international public, 7e éd., Paris, L.G.D.J., 2002 à la p. 583.

7. Michael O'Hanlon, "Transforming NATO: The Role of European Forces" (1997) 39:3 Survival 5 à la p. 13.

8. Bien que des progrès aient été accomplis en matière de politique intérieure concernant l'État de droit, les institutions publiques et la société civile, des lacunes importantes subsistent, notamment dans les domaines de l'application de la loi, du fonctionnement du système judiciaire et de l'indépendance de l'information. La pauvreté est toujours omniprésente et des réformes économiques sont nécessaires. De plus, les problèmes de corruption et de crime organisé n'ont pas été réglés. Robert E. Hunter, "NATO at Fifty: Maximizing NATO: A Relevant Alliance Knows How to Reach" (1999) 78:3 Foreign Affairs 190 à la p. 199. Enfin, les relations tendues que la Russie entretient avec la Tchétchénie ou l'épisode tragique de la prise d'otages de Beslan, montrent que la Russie connaît une fragilité dans le domaine de sa politique intérieure mais elle dispose toutefois toujours de moyens pour influencer la situation de ses voisins aux frontières sud et ouest.

9. Pour les néoconservateurs, les mesures de sécurité collective ont échoué, les organisations internationales sont dominées par des États ennemis des États-Unis et le Conseil de sécurité n'est pas capable de faire respecter ses résolutions. Anatole Lieven, "La marche vers la guerre» (2003) 123 Le Débat 4 à la p. 4-14.

10. En tant que partenaire privilégié des États-Unis, le Royaume-Uni a toujours été particulièrement lié à l'OTAN. L'Allemagne est prise entre son attachement à l'OTAN et son désir de jouer un rôle dans l'élaboration 
la mort de l'Alliance atlantique était donc prématurée. La question est devenue celle de savoir comment transformer une alliance créée il y a 57 ans et dont le ciment était constitué par la volonté collective d'établir un rempart face à l'URSS, en une organisation militaire capable d'affronter les nouvelles menaces que sont le terrorisme international et la prolifération des armes de destruction massive (ADM).

L'Alliance a commencé cette transformation lors de l'adoption, au sommet de Rome du 8 novembre 199111, d'un nouveau concept stratégique ${ }^{12}$. Il était nécessaire d'adapter l'Alliance atlantique à l'ère nouvelle qui s'était ouverte en Europe. Ce concept proposait une approche large de la sécurité, fondée sur le dialogue, la coopération et le maintien d'un potentiel de défense collective. Ce document considérait les difficultés économiques, sociales et politiques, les rivalités ethniques et la prolifération des armes de destruction massive comme de nouvelles menaces et précisait que "la sécurité de l'Alliance doit aussi s'envisager dans un contexte global»13. Il évoquait aussi les deux conclusions à tirer de l'effondrement de l'URSS : "La première, est que la nouveauté

d'une politique européenne de défense. La France, quant à elle, défend plus que tout autre État européen la mise en place d'un système de défense européen indépendant de l'OTAN, mais est consciente de l'importance de cette dernière dans un système de sécurité collective. Ces différentes prises de position ne sont pas nouvelles et le manque d'accord entre les principaux États européens a ainsi freiné la mise en œuvre d'un système concurrent et permis le maintien de l'OTAN. Alyson Bailes, "Europe's Defense Challenge» (1997) 76:1 Foreign Affairs 15 p. 16; KarlHeinz Kamp, "A Global Role for NATO?" (1999) 22:1 The Washington Quarterly 7 à la p. 8.

11. Communiqué final, Déclaration de Rome sur la paix et la coopération, publiée par les chefs d'État et de gouvernement participant à la réunion de Conseil de l'Atlantique Nord, 7 et 8 novembre 1991, "Déclaration de Rome sur la paix et la coopération" (11 mars 2007), en ligne : OTAN <www.nato.int/doc/commf/c9111071.htm>.

12. Les dirigeants alliés y plaidaient pour faire de l'Alliance un "agent du changement" et établissaient une feuille de route pour la transformation de l'OTAN de l'après-guerre froide. L'Alliance a poursuivi ce but avec les sommets de Madrid en 1997, de Washington en 1999, de Prague en 2002 qui sont des étapes de l'adaptation et de la transformation de l'Alliance.

13. "Le Concept stratégique de l'Alliance» (11 mars 2007), en ligne : OTAN <http://www.nato.int/docu/pr/1999/p99-065f.htm>. 
L'OTAN :

vers une conception élargie (2007) 37 R.D.U.S. de la sécurité

de cet environnement n'affecte ni l'objet ni les fonctions de sécurité de l'Alliance, mais en fait ressortir la permanente validité. La seconde, est que ce nouvel environnement offre en revanche à l'Alliance de nouvelles occasions d'inscrire sa stratégie dans le cadre d'une conception élargie de la sécurité».14 Les conclusions adoptées à Rome prévoyaient une dépendance moindre à l'égard des armes nucléaires et des changements concernant les forces intégrées de l'OTAN, comme des réductions de leur taille et de leur niveau de préparation, des améliorations de leur mobilité et un plus large recours aux formations multinationales.

Ces prises de position ont inspiré les changements intervenus dans le concept stratégique de 1991 et la structure des forces ${ }^{15}$. Le dispositif de l'Alliance a été modifié. Il s'agissait de diminuer le volume global des forces, de créer une force de réaction rapide et de faire reposer le dispositif de défense collective sur la formation d'unités multinationales. La rénovation de l'OTAN a aussi conduit au développement de la coopération avec les nouveaux partenaires d'Europe centrale et orientale, par la mise en place de divers appendices et antennes (Partenariat pour la paix ${ }^{16}$, Conseil conjoint permanent OTAN-Russie de 1997 puis le Conseil OTAN - Russie ${ }^{17}$, la Charte OTAN - Ukraine ${ }^{18}$, le Dialogue

14. Ibid.

15. Bob van den Bos, Can Atlanticism Survive? The Netherlands and the New Role of Security Institutions, La Hague, Clingendael Institute, 1992 à la p. 64.

16. L'OTAN a proposé aux anciens membres du Pacte de Varsovie de créer un cadre dans lequel instaurer des relations de confiance par la coopération. Ainsi est né en 1994, le Partenariat pour la paix (PPP), instrument de coopération entre l'OTAN et ses partenaires (Europe centrale et orientale, Caucase du Sud, Asie centrale, pays européens neutres et non alignés : Suisse, Autriche, Finlande, Irlande et Suède), "Le Partenariat pour la paix" (11 mars 2007), en ligne: OTAN <http://www.nato.int>; Hunter, supra note 8 à la p. 193.

17. L'Acte fondateur sur les relations, la coopération et la sécurité mutuelles entre l'OTAN et la Fédération de Russie a été approuvé par le Conseil de l'Atlantique Nord, le 16 mai 1997. Partant du principe que la sécurité de tous les États de la communauté euro-atlantique est indivisible, l'OTAN et la Russie ont décidé de travailler ensemble pour contribuer à l'instauration en Europe, d'une sécurité commune et globale, fondée sur l'adhésion à des valeurs, engagements et normes de comportement 
méditerranéen ${ }^{19}$, le Conseil de partenariat euro-atlantique $(\mathrm{CPEA})^{20}$ qui a succédé au Conseil de coopération nord atlantique $(\mathrm{CCNA})^{21}$ et l'Identité européenne de sécurité et de défense

communs dans l'intérêt de tous les États. Le Conseil conjoint permanent offre un mécanisme de consultation, de coordination, de décision conjointe et d'action conjointe sur les questions de sécurité d'intérêt commun. Acte fondateur sur les relations, la coopération et la sécurité mutuelles entre l'OTAN et la Fédération de Russie, Paris, 27 mai 1997, "Acte fondateur sur les relations, la coopération et la sécurité mutuelles entre l'OTAN et la Fédération de Russie" (11 mars 2007), en ligne: OTAN <www.nato.int/docu/fonda/actfnd-a.htm>.

Le 28 mai 2002, les chefs d'État et de gouvernement des États membres de l'Organisation du Traité de l'Atlantique Nord et de la Fédération de Russie, ont signé une déclaration intitulée "Les relations OTAN-Russie : une qualité nouvelle", par laquelle est créé un nouvel organisme, le Conseil OTAN-Russie. Le Conseil OTAN-Russie constitue, pour les États membres de l'OTAN et la Russie un mécanisme de consultation, d'établissement de consensus, de coopération, de décision et d'action conjointes sur toute une gamme de questions de sécurité dans la région euro-atlantique. "Le sommet OTAN-Russie du 28 mai 2002, une nouvelle donne pour les relations euro-atlantiques?» (11 mars 2007), en ligne: Groupe de recherche et d'information sur la paix et la sécurité $<$ www.grip.org>.

18. La Charte de partenariat spécifique entre l'OTAN et l'Ukraine, signée au sommet de Madrid le 9 juillet 1997, offre aux deux parties de nouvelles occasions de se consulter et de coopérer sur les problèmes politiques et de sécurité. Donald McConnell, "La Charte aidera l'Ukraine à retrouver sa juste place en Europe» (11 mars 2007), en ligne: OTAN <http://www.nato.int>.

19. Lancée fin 1994, l'initiative de l'OTAN en Méditerranée, le Dialogue méditerranéen montre l'importance des questions de sécurité en région méditerranéenne. Le Dialogue méditerranéen n'est pas un programme de coopération militaire, mais un forum politique qui doit permettre de promouvoir l'Alliance atlantique auprès de ces pays, de débattre des questions de sécurité et d'encourager la confiance et la stabilité régionale. Ibid.

20. Le Conseil de partenariat euro-atlantique (CPEA) a remplacé le CCNA en mai 1997. Son but est de lancer une nouvelle phase de coopération. Sergio Balanzino, "Un partenariat plus poussé, clé de la stabilité à long terme en Europe " (1997) $45: 4$ Revue de l'OTAN 10.

21. Le Conseil de coopération nord-atlantique (CCNA) avait pour but de développer le partenariat et la coopération avec les pays d'Europe centrale et orientale, et avait ainsi donné corps au concept d'une communauté euro-atlantique allant de Vancouver à Vladivostok. Bruno 

les pays de l'Est a, certes, compliqué l'organigramme de l'OTAN 23 mais a prouvé aussi que l'OTAN est toujours présente et active sur tous les fronts ${ }^{24}$. En se dotant d'une nouvelle mission de prévention et de gestion des crises, l'OTAN a pu participer aux activités de maintien de la paix en ex-Yougoslavie en 1995 par le biais de l'IFOR puis de la SFOR ${ }^{25}$.

Colson, Europe. Repenser les alliances, Paris, ISC-Économica, 1994, à la p. 124.

22. En 1996, les ministres des Affaires étrangères de l'OTAN réunis à Berlin, ont décidé de développer l'Identité européenne de sécurité et de défense (IESD) au sein de l'Alliance. Cette décision visait à donner aux Alliés européens les moyens de contribuer de façon plus cohérente et efficace aux missions et activités de l'Alliance; de renforcer le partenariat transatlantique et de permettre aux mêmes Alliés européens d'agir seuls, le cas échéant. L'IESD devait symboliser "la création d'un relais stratégique européen au sein même de l'Alliance». Nicole Gnesotto, La puissance et l'Europe, Parsi, Presses de Sciences Po, 1998 à la p. 50.

23. Paul Cornish, "L'OTAN à l'aube du nouveau millénaire : de nouvelles missions, de nouveaux membres... une nouvelle stratégie?», (1997) 45:5 Revue de l'OTAN 21 à la p. 21-24.

24. Pour Bruno Colson, la réforme de l'OTAN initiée en 1991 s'est faite "pour que le leadership américain en ressorte consolidé». Colson, supra note 21 à la p. 136.

25. Les accords de Dayton de 1995 avaient prévu la mise en place d'une force multinationale de mise en œuvre de la paix, créée ensuite par la Résolution 1031 du Conseil de sécurité de l'ONU. Cette force, dénommée Implementation Force (IFOR), composée de 60000 hommes, a été constituée par l'OTAN pour agir sous l'autorité, la direction et le contrôle politique du Conseil de l'Atlantique Nord (...), via la chaîne de commandement de l'OTAN. La mission de l'IFOR, déployée le 20 décembre 1995 dans le cadre de l'opération Joint Endeavour, a d'abord consisté à imposer la cessation des hostilités et à séparer les belligérants. Puis, sous couvert d'un mandat de l'ONU, l'OTAN a décidé la création d'une force de stabilisation (SFOR) pour prendre, par l'opération Joint Guard, le relais de l'IFOR à la fin de l'opération Joint Endeavour, le 20 décembre 1996. Les missions militaires ont pour objet de dissuader toute reprise des combats, d'assurer la protection et la liberté de mouvement de la force, de contrôler et de faire respecter les aspects militaires de l'accord de paix. Il lui revient également de sécuriser l'environnement au profit des organisations civiles, internationales et nationales, d'être à même d'intervenir rapidement sur le théâtre en cas d'incident. La SFOR réunit 35000 hommes, soit la contribution de 15 nations membres de l'OTAN et de 17 États non membres dont la Russie. Comme l'IFOR, la 
Le sommet de Madrid de $1997^{26}$ représente une autre étape majeure de l'histoire de la transformation de l'Alliance, avec l'invitation à entamer des pourparlers d'adhésion à l'OTAN de trois anciens membres du Pacte de Varsovie : la Pologne, la Hongrie et la République tchèque ${ }^{27}$. À Madrid, les chefs d'État et de gouvernement de l'OTAN ont affirmé que l'OTAN devait rester ouverte à de nouveaux membres, conformément à l'article $10 \mathrm{du}$ Traité de l'Atlantique Nord.

De même, l'année 1999 a été marquante pour l'OTAN puisque, d'une part, l'Alliance a confirmé sa prééminence en tant qu'acteur dans la gestion des crises en intervenant au Kosovo en $1999^{28}$ et, d'autre part, elle a adopté un nouveau concept stratégique lors du sommet de Washington ${ }^{29}$. Différents documents ont été approuvés lors du sommet dont le Communiqué final, un document politique qui a défini le cadre des relations entre l'OTAN et l'UE. Le nouveau Concept stratégique a aussi défini la mise en œuvre de la stratégie de l'Alliance sur le plan militaire et fixe les missions militaires de l'Alliance, notamment la nouvelle mission de gestion des crises. Le Plan d'action pour l'adhésion des nouveaux pays candidats, quant à lui, a précisé les conditions à remplir pour adhérer à l'Alliance. Enfin, l'Initiative sur les capacités de défense a cherché à donner à l'Alliance les capacités militaires nécessaires qui lui font défaut pour la gestion des crises.

SFOR agit sur décision du Conseil de l'Atlantique Nord. William R. Phillips, "La coopération civilo-militaire, élément essentiel à la mise en œuvre de la paix en Bosnie» (1998) 46 : 1 Revue de l'OTAN 22 à la p. 2225.

26. "Déclaration de Madrid sur la sécurité et la coopération euro-atlantiques» (11 mars 2007), en ligne : OTAN <http://www.nato.int>.

27. O’Hanlon, supra note 7 à la p. 5 .

28. "Déclaration sur le Kosovo" (11 mars 2007), en ligne: OTAN $<$ http://www.nato.int>.

29. "Déclaration de Washington" (11 mars 2007), en ligne: OTAN <http://www.nato.int>; Karl-Heinz Kamp, "L'OTAN après le Kosovo : ange de paix ou gendarme du monde ?» (1999) 64 : 2 Politique étrangère 245 aux p. 246-251. 
Après avoir rempli avec succès la mission pour laquelle elle avait été créée, l'Alliance s'est donc trouvée confrontée à de nouveaux risques et défis dans un environnement sécuritaire international en constante évolution. D'organisation de défense lors de sa création, l'OTAN a réussi à se transformer en une organisation de défense et de sécurité collective en 199930 puis à une organisation antiterroriste ${ }^{31}$. Les États-Unis sont les principaux acteurs de l'évolution de l'Alliance.

Selon eux, l'OTAN ne devrait pas uniquement servir à défendre l'intégrité territoriale de ses membres, mais également à lutter contre le terrorisme et à mener des interventions humanitaires en dehors de son territoire. Bien que la défense collective demeure l'objectif premier de l'OTAN, réaffirmé lors de chaque sommet, les missions relevant de cette responsabilité sont très différentes de celles que l'Alliance avait prévues pendant la Guerre froide ${ }^{32}$. Les opérations conduites en Bosnie-Herzégovine et au Kosovo ont en effet déplacé le centre de gravité de l'OTAN de la défense collective vers la gestion des crises et elles ont permis à l'Organisation atlantique de s'affranchir du dogme de la zone de

30. La première intervention de l'OTAN dans le domaine de la sécurité collective est apparue dans le communiqué final de la réunion ministérielle d'Oslo, le 4 juin 1992. Les Alliés se déclaraient prêts à soutenir "les activités de maintien de la paix entreprises sous la responsabilité de la CSCE, y compris en mettant à disposition les ressources et les compétences de l'Alliance". "Communiqué Final" (11 mars 2007), en ligne : OTAN <http://www.nato.int/docu/ commf/c9206041.htm>. Le sommet de Washington du 24 avril 1999 a marqué une étape importante dans la rénovation de l'Alliance. Le nouveau concept stratégique adopté à cette occasion a défini la mise en œuvre de la stratégie de l'Alliance sur le plan militaire et fixé les nouvelles missions de gestion des crises. Javier Solona, "Un moment déterminant pour l'OTAN : les décisions du sommet de Washington et la crise du Kosovo" (1999) 47 : 2 Revue de l'OTAN 3 à la p. 4.

31. L'OTAN a mené l'opération Active Endeavour qui est l'une des premières opérations contre le terrorisme. Des navires et des avions alliés contribuent à la guerre contre le terrorisme en effectuant des patrouilles maritimes en Méditerranée et des arraisonnements autorisés de navires présumés terroristes. "Opération Active Endeavour" (11 mars 2007), en ligne : OTAN <http://www.nato.int>.

32. Ian Brzezinski, "L'OTAN : une alliance en cours de transformation" (11 mars 2007), en ligne: U.S, Department of State <www.usinfo.state.gov>. 
responsabilité et de compétence. Les contributions des forces alliées aux opérations militaires menées dans les Balkans, en Méditerranée, en Afghanistan et en Irak témoignent aussi des responsabilités mondiales de plus en plus lourdes qui incombent à l'OTAN33.

Pour les assumer, l'OTAN s'est lancée dans un processus de transformation et de renouvellement. Cette évolution s'est clairement dessinée au sommet de Washington et s'est poursuivie au sommet de Prague de novembre 2002. La transformation de l'Alliance induit une refondation complète de ses structures. Elle représente une nouvelle vision pour l'OTAN et un bouleversement par rapport à l'objectif essentiel original de l'Alliance. La menace ayant changé, il est devenu nécessaire de restructurer les forces armées de l'Alliance et comme le souligne l'ancien secrétaire général de l'OTAN, Lord Robertson: "Nous transformons notre Alliance afin qu'elle reste un élément central de la lutte contre les menaces du XXIe siècle, en particulier le terrorisme et les armes de destruction massive» ${ }^{34}$. La mise en place de cette nouvelle OTAN s'est faite par étapes successives. Le processus de transformation entamé depuis les années 90 a connu deux moments déterminants avec les sommets de Prague en 2002 et d'Istanbul en 2004, qui ont mené à la mise en place de la Force de réaction de l'OTAN (NRF) et d'une nouvelle structure de commandement. C'est en grande partie grâce à ces deux innovations que l'OTAN est maintenant en mesure, tel qu'il en sera discuté dans la partie II de l'article, d'affronter le nouvel environnement de sécurité et de mener de nouveaux types de missions.

\section{La mise en place de la NRF}

Décidée lors du sommet de Washington en 1999, la transformation de l'Alliance s'est poursuivie et adaptée lors du

33. Virginie Sandrock, Aude Le Calve, "Les clefs de l'OTAN" (2005) 297 Armées d'aujourd'hui 36.

34. Discours de Lord Robertson au Sommet informel de l'OTAN à Varsovie, le 24 septembre 2002, http://www.shape.nato.int/docu/update/2002/09september/f0924a.htm. 
sommet de Prague ${ }^{35}$. Elle induit une refondation complète de ses structures, qui passe par la mise en place d'un nouveau concept : la Force de réaction de l'OTAN (FRO ou Nato Response Force NRF).

Le sommet de Prague a posé les jalons de plusieurs initiatives décisives en matière de transformation militaire. Les enjeux du sommet de Prague étaient l'invitation pour sept États ${ }^{36}$ d'entamer des négociations d'adhésion ${ }^{37}$, l'acquisition de nouvelles capacités, et la création de nouveaux partenariats et le renforcement de ceux existants ${ }^{38}$. L'une des décisions les plus notables de ce sommet fut celle des chefs d'État et de gouvernement de créer une force de réaction de l'OTAN (NRF). Cette création correspond à l'un des objectifs du processus de transformation initié à Prague. La NRF est, à l'origine, une proposition émanant de l'administration américaine, présentée lors d'une réunion informelle des ministres de la défense de

35. Lionel Crochard, "Le Sommet de l'OTAN, Prague 2002" (2003) 4 Défense nationale et sécurité collective 8 .

36. Il s'agit de la Bulgarie, l'Estonie, la Lettonie, la Lituanie, la Roumanie, la Slovaquie et la Slovénie. L'adhésion de l'Albanie et de la Macédoine a été repoussée à une date ultérieure.

37. Avec l'arrivée des nouveaux membres, la population de l'Alliance passe de 735 millions à 839 millions (soit un accroissement de 14\%), les forces armées d'active augmentent de 16\%; les contingents de réserve augmentent considérablement; les PECO apportant 1714700 hommes supplémentaires (soit un accroissement de 45\%); le budget militaire de l'Alliance n'enregistrera lui, que 1,5\% de hausse. Peter Sandor, "Prague : le sommet de la transformation de l'OTAN" (11 mars 2007), en ligne: Synthèse $n^{\circ} 69$ <www.robert-schuman.org/synth69.htm>.

38. Au sommet de Prague, l'OTAN a cherché à approfondir et à renforcer ses partenariats dans le cadre du Conseil de partenariat euro-atlantique (CPEA) qui constitue le cadre général de la coopération de l'OTAN avec ses partenaires d'Europe centrale et orientale; du Caucase et d'Asie centrale; de la Charte OTAN-Ukraine; du Dialogue méditerranéen en élargissant les dimensions politiques et pratiques; du partenariat OTANUnion européenne sur des questions d'intérêt commun liées à la sécurité, la défense et la gestion des crises et du Conseil OTAN-Russie. Alberto Bin, "Le renforcement du Dialogue méditerranéen de l'OTAN" (11 mars 2007), en ligne: Revue de l'OTAN <http://www.nato.int/docu/review/ 2003/issue 1/french/art4.html>. 
l'Alliance à Varsovie, le 22 septembre $2002^{39}$ et adoptée par les chefs d'État et de gouvernement des pays membres, au sommet de Prague. Ce projet s'inscrit dans la stratégie adoptée par l'administration américaine, à la suite des événements du 11 septembre 2001, de combattre à l'échelle planétaire le terrorisme et la menace des armes de destruction massive et de terreur, détenues ou en voie de l'être par des Etats "voyous". La stratégie de sécurité nationale prévoit la mise à disposition d'outils militaires adéquats, rapides, flexibles et interarmées, tels que la NRF.

Pendant la Guerre froide, l'Alliance se concentrait sur la masse et la puissance de feu. Mais les menaces actuelles nécessitent des forces souples, proactives et manœuvrables. Pour cela, les Alliés doivent poursuivre la réforme de leurs armées afin de pouvoir les mettre à disposition de la NRF pour des missions pour lesquelles celles-ci devront être rapidement déployées dans n'importe quelle région du monde, et assurer leur capacité de soutien sur le terrain. La NRF correspond au besoin de l'OTAN de disposer d'une force interarmées multinationale hautement performante, capable de réagir à très bref délai.

La NRF a pour objectif de permettre à l'Alliance de faire face aux nouvelles menaces du XXIe siècle, et de pouvoir réagir à l'intérieur comme à l'extérieur de la zone d'intervention de l'Alliance ${ }^{40}$. La NRF doit être : "souple, déployable, interopérable et

39. Sommet informel de l'OTAN à Varsovie, le 24 septembre 2002, "Réunis à Varsovie, les ministres de la défense centrent leur réflexion sur la transformation de l'OTAN" (11 mars 2007), en ligne: OTAN $<$ http://www.nato.int>.

40. Le Général Haral Kujat, président du Comité militaire de l'OTAN, lors d'un séminaire de l'OTAN, Open Road, organisé au SACLANT (Norfolk, États-Unis) en janvier 2003, a souligné, que "[...] la Force de réaction de l'OTAN est le vecteur privilégié sur lequel devra se concentrer le processus de transformation, car cette innovation fournira à l'OTAN une arme équilibrée, apte à exécuter toute la gamme de ses missions. Elle constitue à ce titre le point de ralliement pour tous les membres de l'Alliance et c'est ainsi qu'elle doit être perçue. Mais notre objectif ne s'arrête pas à la FRO. Nous devons transformer toutes nos forces et leurs capacités. Les États-Unis doivent être présents sur un pied d'égalité avec 
L'OTAN :

vers une conception élargie $\quad$ (2007) 37 R.D.U.S. de la sécurité

apte à soutenir des opérations prolongées et qui comportent des éléments terrestres, maritimes et aériens prêts à se transporter rapidement partout où il le faudra", selon la décision du Conseil.41. La Force de réaction de l'OTAN doit servir de catalyseur permettant de cibler et d'améliorer les capacités militaires de l'Alliance. Elle s'intègre dans le processus de transformation des structures militaires de l'Alliance, en cours depuis les années 1990 et rendu indispensable par les changements stratégiques et géopolitiques intervenus en Europe, tels que l'évolution de la situation en Russie et les nouvelles zones d'instabilité dans les Balkans.

Cette force 42 , dont l'effectif est estimé à 21000 soldats, se compose tout d'abord de moyens aériens qui doivent pouvoir gérer au moins 200 sorties par jour, en assurant une capacité de projection rapide, en menant des missions aériennes classiques telles que des missions de combat, de surveillance, d'interdiction ou de ravitaillement et des missions de transport stratégique. Ensuite, la composante terrestre doit permettre le déploiement d'une force de la taille d'une brigade (5 000 soldats environ), capable d'exécuter tous types de missions terrestres dans un grand nombre de scénarios et de localisations géographiques ${ }^{43}$.

leurs partenaires dans le processus de transformation de l'Alliance. Cela implique naturellement qu'ils doivent participer à son noyau commun, la FRO, mais aussi à chacune des facettes du processus de transformation. Autrement dit, ils doivent faire partie du couteau de l'OTAN, de son manche et de sa lame [...]" "Séminaire de haut niveau sur la transformation militaire de l'OTAN" (11 mars 2007), en ligne : OTAN <http://www.nato.int/docu/update/2003/01-january/f0120a.htm>.

41. "Nous avons donné des instructions pour que soit élaboré un concept global d'emploi de cette force, qui atteindra sa capacité opérationnelle initiale dès que possible, mais au plus tard en octobre 2004, et sa capacité opérationnelle finale au plus tard en octobre 2006". "Déclaration du sommet de Prague, les 21 et 22 novembre 2002" (11 mars 2007), en ligne: OTAN <http://www.nato.int>.

42. "La Force de réaction de l'OTAN : au centre de la transformation de l'OTAN" (11 mars 2007), en ligne : OTAN <http://www.nato.int>.

43. Cette composante doit inclure des éléments lourds, légers et aéroportés. Les moyens sont très divers et incluent l'artillerie, le soutien aérien, le génie, les forces spéciales, la logistique et la manutention, les communications, la défense antiaérienne, la défense contre la menace 
Enfin, les forces navales de la taille maximale d'une force navale permanente de l'OTAN ${ }^{44}$, doivent conduire une gamme variée de missions maritimes telles que le transport stratégique, l'escorte, la lutte anti-sous-marine et anti-mine, la défense antiaérienne et l'attaque à terre. Cette force peut également être une composante initiale d'un dispositif du niveau d'un corps d'armée (soit 100000 hommes) appelé HRF (High Readiness Force).

La Force de réaction est composée des meilleures unités nationales, les mieux équipées et les mieux entraînées ${ }^{45}$. C'est une force interarmées, entraînée et "certifiée" O.T.A.N. ${ }^{46}$, dont le degré de disponibilité et de préparation est élevé, et qui peut agir de manière autonome ou préparer l'arrivée d'autres forces. Elle doit disposer de sa propre logistique, de capacités de commandement et de contrôle, être capable de rester sur le terrain au moins 30 jours (en attendant le ravitaillement ultérieur) et aussi d'agir en tant que force de combat offensive. Un niveau élevé de préparation nécessite que les personnels soient très bien entraînés, bien équipés, prêts à intervenir et à partir rapidement en cas de crise.

chimique, biologique, radioactive et nucléaire, le transport, les capacités civilo-militaires, la police militaire, les services sanitaires et l'information. "Exercice de déploiement de la Force de réaction de l'OTAN" (11 mars 2007), en ligne : OTAN <http://www.nato.int>.

44. La force navale opérationnelle doit inclure un groupe aéronaval complet c'est-à-dire des unités de combat, de surface ou sous-marines, des forces amphibies, des forces antimines et de soutien. "Briefing: améliorer les capacités pour répondre à de nouvelles menaces" (11 mars 2007), en ligne : OTAN <http://www.nato.int>.

45. Au niveau des unités terrestres, de grandes unités européennes ont été déclarées disponibles tant pour l'OTAN que pour l'UE comme le Corps de réaction rapide du Commandement allié en Europe (ARRC), essentiellement britannique, le Corps européen (Eurocorps) et le Corps germano-néerlandais. "L'Eurocorps se prépare pour la NRF 7" (11 mars 2007), en ligne : mil.be <http://www.mil.be/def/news/ index.asp?LAN= fr\&ID=391>.

46. Des normes et procédures pour la certification de la NRF ont été établies. Elles fixent des critères pour la certification, la soutenabilité à long terme et l'amélioration de la qualité générale, des capacités et de l'état de préparation de la NRF. 
La NRF est un moyen de doter l'OTAN de capacités spéciales ultrasophistiquées destinées à l'ensemble des missions de l'Alliance ${ }^{47}$, les Alliés fournissant des forces en vertu d'un système de rotation de six mois. Après s'être entraînée pendant cette période, la NRF reçoit une certification aux normes les plus élevées, particulièrement en ce qui concerne les capacités et l'interopérabilité. Elle est alors disponible sur appel pendant six mois. Ces éléments de force sont ensuite remplacés par de nouvelles troupes fournies sur une base volontaire, qui formeront un nouveau prototype. Ces troupes suivent un programme d'entraînement semblable et sont disponibles sur appel, pour la même période, avant d'être remplacés par d'autres forces une fois le cycle terminé. Tous les pays membres de l'OTAN souscrivent à ce système de rotation qui apporte des avantages majeurs aux troupes concernées, en leur fournissant l'expérience d'un entraînement spécial et l'intégration dans une force dont l'état de préparation répond aux normes les plus élevées ${ }^{48}$. Le lancement de la NRF est accompagné par une nouvelle initiative en matière d'équipements, l'Engagement capacitaire de Prague ${ }^{49}$.

47. Lors de la réunion ministérielle du Comité des plans de défense de l'OTAN, les ministres de la Défense se sont réjouis du fait que la plupart des Alliés restructurent "[...] leurs forces pour en réduire la taille et les rendre plus souples, plus modernes et plus efficaces pour la gamme complète des missions de l'Alliance". Toutefois, alors que, pour la première fois depuis longtemps, une grande partie des Alliés prévoit d'accroître ou de maintenir le financement de la défense, les budgets de la Défense ne permettent toujours pas de répondre aux besoins essentiels de restructuration et de modernisation des armées nationales. "Communiqué final: Réunion du Comité des plans de défense et du Groupe des plans nucléaires en session ministérielle tenue à Bruxelles le 12 juin 2003" (11 mars 2007), en ligne : OTAN <http://www.nato.int>.

48. "La force de réaction de l'OTAN; Au centre de la transformation de l'OTAN" (11 mars 2007), en ligne : OTAN <http://www.nato.int> [La force de réaction de l'OTAN].

49. Les chefs d'État et de gouvernement de l'Alliance ont décidé, lors du sommet de Prague de décembre 2002, d'un ensemble d'engagements intitulé "l'Engagement capacitaire de Prague», dans 8 domaines couvrant 400 besoins plus spécifiques : la défense contre les attaques chimiques, biologiques, radiologiques et nucléaires (CBRN) - la surveillance air/sol le commandement, le contrôle et les communications - le renseignement, 
L'Alliance a inauguré la première force de rotation NRF prototype, la "NRF 1", au quartier général régional nord de Brunssum aux Pays-Bas, le 15 octobre 2003. Le premier prototype de la Force a réuni 8500 hommes. La NRF 2 a pris le relais en janvier 2004, en restant sous le commandement de l'AFNORTH (Forces Alliées du Nord Europe). Les deux premières rotations NRF opérationnelles restaient expérimentales. Elles ont été conçues pour être d'une ampleur limitée. Quatre prototypes de rotation ont déjà été mis en activité. Ils ont été utilisés pour mettre le concept à l'essai et régler les détails pratiques de fonctionnement de la Force. Avant d'avoir été prêtes à exécuter la gamme complète d'opérations, les NRF 3 et 4 avaient déjà atteint la capacité opérationnelle. Ces forces se sont entraînées en participant à des exercices comme Allied Response, organisés à Doganbey en Turquie ${ }^{50}$, en novembre 2003 et Allied Action en Italie en mai 200451. L'exercice Allied Warrior 2004 s'est tenu à Amersfoort, Ede

la surveillance et l'acquisition d'objectifs - l'efficacité au combat et notamment, les munitions à guidage de précision et la neutralisation des défenses aériennes ennemies - le transport aérien et maritime stratégique - le ravitaillement en vol - les unités déployables d'appui tactique et de soutien des forces au combat. "L'OTAN après Prague" (11 mars 2007), en ligne : OTAN <http://www.nato.int>. .

50. Le premier exercice militaire a vu la participation de plus de 1000 soldats venus de 12 pays membres. La force, créée le 15 octobre 2003, regroupe notamment l'Allemagne, la Belgique, le Danemark, l'Espagne, la France, la Grèce, la Norvège, la Pologne, la République tchèque, le Royaume-Uni et la Turquie. L'exercice visait à montrer la capacité de la force dans un exercice d'entraînement en plein air impliquant un mandat onusien et une opération de réaction de l'OTAN. "OTAN" (11 mars 2007), en ligne: Fondation Robert Schuman <http://www.robertschuman.org/lettre/lettre144.htm\#19>.

51. Lors de l'exercice Allied Action 04 qui s'est déroulé du 25 mai au 8 juin 2004, l'OTAN a procédé pour la première fois à un essai d'activation du quartier général d'une force opérationnelle interarmées déployable (DJTF), ce qui a constitué un entraînement en vue des opérations de la force de réaction de l'OTAN. L'exercice Allied Action 04 a été l'occasion pour les participants, de mettre en pratique l'activation et le déploiement de la Force de réaction de l'OTAN, afin de se préparer à la conduite des opérations de suivi d'un groupe de forces interarmées multinationales (GFIM). Cet exercice était avant tout un exercice de poste de commandement d'un GFIM. Plus de 2600 soldats de 23 pays membres de l'OTAN et de 12 pays partenaires participent à l'exercice. "Briefing : La 
et Harskamp aux Pays-Bas et à Vérone et Naples en Italie, du 8 au 24 novembre 2004 et a rassemblé plus de 900 participants de 16 pays de l'OTAN52. Cet exercice concernait les procédures de déploiement et de commandement de la Force de réaction de l'OTAN pour les missions de gestion des crises. La doctrine, l'organisation, l'entraînement, les procédures de certification et les fonctions de commandement et de contrôle font partie des aspects qui ont été évalués au cours de cette première période d'essai. La $\mathrm{NRF}$ a ainsi atteint sa capacité opérationnelle initiale de 17000 hommes et a été déclarée prête à remplir toute la gamme de ses missions à l'endroit et au moment où l'Alliance décidera de l'utiliser. L'annonce en a été faite le 13 octobre 2004 en Roumanie, par le secrétaire général de l'OTAN, M. Jaap de Hoop Scheffer et par le Commandant suprême des forces alliées en Europe (SACEUR), le général James L. Jones, lors de la réunion informelle des ministres de la Défense des pays de l'OTAN53.

D'autres exercices d'entraînement ont eu lieu. Les exercices Allied Action 200554, qui se sont déroulés au Centre de guerre interarmées de Stavanger en Norvège, et Allied Warrior 200555,

Force de réaction de l'OTAN" (11 mars 2007), en ligne: OTAN <http://www.nato.int/docu/briefing/nrf2006/html_fr/nrf03.html>.

52. L'exercice mettait en scène la menace d'une attaque asymétrique à l'aide d'armes nucléaires, biologiques et chimiques nécessitant l'intégration des capacités de soutien du bataillon OTAN multinational de défense contre les armes chimiques, biologiques, radiologiques et nucléaires (CBRN) et de l'équipe d'évaluation conjointe NBC. Il concernait essentiellement le poste de commandement. "Nato, esercitazione Allied Warrior 2004" (11 mars 2007), en ligne: Pagine di difesa <http://www.paginedidifesa.it/ 2004/pdd_041113.html>.

53. "La Force de réaction est prête" (5 juillet 2007), en ligne : OTAN <http://www.nato.int/docu/update/2004/10-october/f1013a.htm>.

54. L'exercice Allied Action 05 s'est déroulé du 17 mai au 2 juin 2005. C'était une véritable étape finale pour la certification des structures de la NRF. L'exercice devait permettre d'affiner les capacités de déploiement et de direction d'une composante aérienne. «31/05/05 - Allied Action 05 : en route vers la NRF5" (11 mars 2007), en ligne : Ministère de la défense <http://www.defense.gouv.fr/air/base/breves/2005/ mai_05/31_05_05_allied_action_05_en_route_vers_la_nrf5 >.

55. «Briefing : Déployer les forces plus rapidement et plus loin que jamais» (11 mars 2007), en ligne : OTAN <http://www.nato.int>. 
sont des exercices de certification, respectivement pour l'ensemble de la NRF 5 et pour le commandement de la composante terrestre de la NRF 6. La NRF a aussi testé ses capacités à conduire des opérations complexes en mer dans un exercice amphibie de l'OTAN, baptisé Destined Glory (Loyal Midas), en mer Tyrrhénienne, du 29 septembre au 14 octobre 2005. Huit mille cinq cents militaires, 37 bateaux et 57 avions provenant de dix pays membres de l'OTAN ont pris part à cet exercice. Le but était de tester et de certifier les forces qui devaient constituer la composante maritime de la NRF de janvier à juin 200656 . L'exercice intitulé Steadfast Jaguar ${ }^{57}$ qui s'est déroulé aux îles du Cap-Vert, a associé pour la première fois les composantes terrestre, maritime et aérienne de la NRF et s'est achevé avec succès en juin 2006. Les besoins en forces interarmées multinationales portant sur les capacités et unités requises pour la septième rotation de la NRF58 au cours de la première moitié 2007 sont aussi satisfaits par les États membres.

Les principales lacunes observées lors de la mise en place des premières rotations de la NRF sont celles concernant le transport stratégique aérien et la protection contre les armes nucléaires, biologiques et chimiques. La NRF doit aussi faire face à plusieurs défis: processus de prise de décision, planification précoce, partage de l'information, inclusion de forces de pays nonOTAN dont les pays partenaires. Lors de la réunion du Comité des plans de défense et du Groupe des plans nucléaires du 9 juin 2005, les ministres de la Défense des États membres de l'OTAN ont réaffirmé leur volonté de mener la NRF jusqu'à sa capacité

56. L'exercice a présenté des scénarios complexes incorporant des situations inattendues. "In previous years, [the commander] has had a program to go by; this year he [didn't], stressed Rear Adm. Cooke, Deputy Commander of Naval Striking and Support Forces NATO». La force de réaction de l'OTAN, supra note 48.

57. "Exercise Steadfast Jaguar 06: the NRF concept test" (11 mars 2007), en ligne: OTAN <http://www.nato.int/shape/issues/shape_nrf/ steadfast_jaguar.htm>.

58. La rotation de la NRF 8 se déroulera de janvier à mi-juillet 2007. «Briefing : La Force de réaction de l'OTAN : 5. Les rotations de la Force de réaction de l'OTAN" (11 mars 2007), en ligne: OTAN <http://www.nato.int/docu/briefing/nrf2006/html_fr/nrf05.html> 
opérationnelle finale. Conscients que les exigences imposées sont importantes, les pays de l'OTAN ont néanmoins réaffirmé leur détermination à mener à bien la transformation en cours des capacités de l'Alliance et "à remédier aux insuffisances qui subsistent dans certains domaines de capacités essentiels»59.

À mesure que les différentes forces qui se succèdent au sein de la NRF appliquent ces normes élevées, le processus de transformation s'étend peu à peu à toutes les forces alliées. Reposant sur un cycle d'alerte triennal, il est prévu que le commandement de la NRF sera placé à tour de rôle sous le commandement de l'état-major de l'AFNORTH (Forces alliées du Nord Europe) à Brunssum (Hollande); de celui des forces interarmées de Naples (Italie) et enfin de celui du QG interarmées de Lisbonne (Portugal) lors des rotations futures. La NRF dispose ainsi de trois structures de commandement interarmées assurant la mise sur pied, la préparation opérationnelle et l'engagement éventuel de cette force. Le transfert d'autorité au SACEUR (Commandement suprême des forces alliées en Europe) doit avoir lieu une fois qu'aura été prise la décision politique nécessaire au déploiement de la Force de réaction de l'OTAN pour une opération spécifique.

Bien que la NRF n'a atteint sa pleine capacité opérationnelle que fin 2006, elle a déjà été déployée pour une première opération. Alors que ce sont les États-Unis qui ont souhaité développer la NRF afin que l'OTAN puisse intervenir dans le cadre, notamment d'une opération civilo militaire, ce sont eux qui vont bénéficier de la première opération de la NRF, justifiant ainsi la vision qu'ils ont voulu inculquer à l'évolution de l'Alliance. En effet, après que l'ouragan Katrina a frappé les États de

59. Les lacunes se trouvent principalement au niveau des capacités facilitatrices essentielles. Par conséquent, le déséquilibre entre les forces de combat et les forces de soutien déployables limite la capacité de certains pays à déployer des unités et à en assurer le soutien dans le cas d'opérations exigeantes, menées loin du territoire de l'OTAN. "Communiqué final; Réunion du Comité des plans de défense et du Groupe des plans nucléaires en session ministérielle" (11 mars 2007), en ligne : OTAN <http://www.nato.int/docu/pr/2005/p05-075f.htm>. 
l'Alabama, de Floride, de Louisiane et du Mississippi le 28 août 2005, le Centre euro-atlantique de coordination des réactions en cas de catastrophe (EADRCC) de l'OTAN a offert ses services aux États-Unis. Le 8 septembre 2005, le Conseil de l'Atlantique Nord a autorisé une opération de transport de l'OTAN, composée d'avions de formation et de transport, de la Force aéroportée de détection lointaine et d'avions et de navires de transport de la Force de réaction de l'OTAN (NRF), afin de contribuer à l'acheminement urgent de matériel depuis l'Europe vers les États-Unis. L'EADRCC a rempli le rôle de bureau de centralisation entre les demandes et les offres d'aide. La NRF est intervenue par un pont aérien tactique placé sous l'autorité du Commandement conjoint à Lisbonne. Cette première intervention fut certes circonscrite, mais le symbole était là: les États-Unis, conducteurs de la transformation de l'OTAN en une organisation de sécurité, démontraient la nécessité de celle-ci.

La deuxième intervention de la NRF fut encore plus anecdotique de par son ampleur. Deux jours après le tremblement de terre qui a dévasté la région du Cachemire, le 8 octobre 2005, le Pakistan a demandé l'aide de l'OTAN pour l'opération de secours humanitaire qu'il était en train de mettre sur pied. L'intervention de l'OTAN se fit en deux phases. Lors de la première phase, le Centre euro-atlantique de coordination des réactions en cas de catastrophe (EADRCC) a établi des liens entre les instances nationales de coordination de l'aide fournie par ses différents membres et les autorités pakistanaises. La NRF est intervenue lors de la seconde phase de l'opération, en assurant le pont aérien et en fournissant un quartier général responsable de la structure de commandement et de contrôle, des unités du génie, des hélicoptères et des hôpitaux de campagne militaires. Mais il faut souligner que la plus grande partie de l'aide fut fournie par des forces traditionnelles.

On peut alors se demander quelle est la valeur ajoutée de l'OTAN, en général, et de la $\mathrm{NRF}$ en particulier dans ces opérations. La principale qualité de l'OTAN est de remplir un rôle de coordination et de liaison grâce notamment à l'EADRCC. Les structures de l'Alliance permettent notamment à des Alliés de 
fournir des capacités, comme des unités médicales, qu'ils n'auraient pu acheminer seuls. Le second avantage de l'OTAN est sa capacité à s'organiser rapidement dès lors qu'il y a déjà eu un précédent et que les procédures ont déjà été testées. Ainsi, la mise en place du pont aérien vers le Pakistan a été facilitée du fait de l'expérience de Katrina. En définitive, si les expériences de la NRF sont pour l'instant très limitées et ne répondent pas encore aux objectifs qu'on lui avait assignés, elles méritent, pour le moins, d'exister et de structurer l'action collective des membres.

Dans le même temps, l'Union européenne a, elle aussi, décidé de mettre en place une force de réaction rapide ${ }^{60}$. Les deux forces ont vu le jour avec très peu de différence : 2003 pour la mise en place finale des capacités d'intervention rapide de l'UE et 2004 pour la constitution de la NRF. Dans les deux cas, ce n'est pas seulement une question technique de capacités militaires, c'est l'avenir même de l'UE et de l'OTAN, en tant qu'organisations de sécurité et de défense européenne et transatlantique, qui est l'enjeu majeur. Le respect des engagements souscrits et du calendrier prévu, ainsi que l'établissement d'une réelle coopération entre les deux organisations, pour éviter la duplication et les divisions entre Européens, sont nécessaires pour l'efficacité des deux dispositifs. De plus, la complémentarité des deux organisations est nécessaire dans le cadre de la gestion des crises.

Les deux forces ne peuvent faire appel qu'aux mêmes réservoirs de forces nationales ${ }^{61}$, dont certaines sont maintenues en alerte pour être projetables sous faible préavis ${ }^{62}$. Cependant, seul un petit noyau d'unités très réactif est nécessaire pour la $\mathrm{NRF}$, puisqu'il s'agit de déployer très rapidement une brigade,

60. "Conseil européen d'Helsinki" (11 mars 2007), en ligne : Europa <http://europa.eu.int/council/off/conclu/dec99/dec99_fr.htm>.

61. C'est un problème pour les États qui sont membres à la fois de l'OTAN et de l'UE.

62. Au niveau des unités terrestres, de grandes unités européennes sont capables de déploiement rapide et ont été déclarées disponibles, tant pour l'OTAN que pour l'UE comme le Corps de réaction rapide du Commandement allié en Europe, essentiellement britannique, le Corps européen et le Corps germano-néerlandais. 
dotée d'éléments de combat et de soutien, alors que l'UE doit disposer, outre ce noyau réactif constitué à partir des mêmes unités, d'une large panoplie d'unités pour mettre en place une force de 50 à 60000 hommes, adaptée à la mission. Dans certains cas, on retrouvera les mêmes unités dans l'objectif global de l'UE et dans la NRF. Les différents exercices qui ont déjà eu lieu pour aider la NRF d'être opérationnelle, permettent en même temps aux forces nationales de se former et de renforcer leur complémentarité. En effet, puisque l'on retrouve dans le cadre de la NRF, les principaux États contributeurs de la Force de réaction rapide de l'UE, les différentes opérations organisées ont aussi permis de renforcer la capacité européenne de gestion des crises et d'intervention militaire en fournissant à l'UE un ensemble de forces entraînées ${ }^{63}$.

En application des arrangements "Berlin Plus»64, l'OTAN pourrait mettre, sur demande du Conseil de l'Union européenne,

63. La France et la Grande Bretagne sont les principaux contributeurs de la NRF. Le partenariat franco-britannique se caractérise par une contribution de près de $80 \%$ des personnels de la structure de commandement et de conduite air (JFACC) et d'environ $40 \%$ des moyens aériens; les autres nations de l'OTAN fournissant les moyens complémentaires. "La composante aérienne de la cinquième Nato Response Force (NRF 5)" (11 mars 2007), en ligne: Ministère de la défense <http://www.defense.gouv.fr/defense/content/download/ 47866/474995/file/la_composante_aerienne_de_la_cinquieme_nato_res ponse_fo_nrf.pdf $>$.

64. Les arrangements "Berlin Plus", mis en place en 1996 lors de la réunion au cours de laquelle les ministres des Affaires étrangères des pays de l'OTAN ont décidé de créer une Identité européenne de sécurité et de défense, visent à éviter les doubles emplois et comportent quatre éléments : la garantie de l'accès de l'UE à des capacités de planification opérationnelle de l'OTAN; la présomption de disponibilité au profit de l'UE de capacités et de moyens communs de l'OTAN; des options de commandement européen pour des opérations dirigées par l'UE, y compris un renforcement du rôle de l'adjoint au Commandant suprême des forces alliées en Europe (SACEUR) et l'adaptation du système de planification de la défense de l'OTAN pour y intégrer la disponibilité de forces pour des opérations de l'UE. "EU-NATO: The framework for permenent relations and Berlin plus" (11 mars 2007), en ligne : Conseil de l'Union Europpéenne <http://www.consilium.europa.eu/uedocs/ cmsUpload/03-11-11\%20Berlin\%20Plus\%20press\%20note\%20BL.pdf>. 
la NRF ou d'importants éléments de cette force à la disposition de l'UE. Il est donc important que ces forces utilisent des normes et des procédures similaires pour éviter le double emploi. L'entraînement de la NRF et des groupes tactiques de l'UE ensemble, lors d'exercices coordonnés, doit donc être encouragé. De plus, le caractère rapide de la $\mathrm{NRF}$ et des forces de l'objectif global de l'UE, exige aussi la mise en place et le développement de mécanismes politiques de prise de décision et d'un dialogue permanent entre l'Alliance et l'UE, pour éviter que les actions de l'une de ces organisations ne privent l'autre d'une partie de ses moyens.

Même si la NRF doit accomplir toutes les missions de l'Alliance, les siennes seront principalement celles qui exigent une capacité de réagir en déployant les forces les plus performantes dans un délai très bref. Il pourra s'agir d'une part, de se déployer en tant que force autonome pour des opérations relevant de l'article $5 \mathrm{du}$ Traité de l'Atlantique Nord (défense collective) et des opérations de réponse aux crises hors article 5, telles que des opérations d'évacuation, des opérations à l'appui de la gestion des conséquences d'une catastrophe (notamment des incidents chimiques, biologiques, radiologiques et nucléaires), des situations de crise humanitaire et des opérations de lutte contre le terrorisme. Il pourra s'agir d'autre part, de se déployer en tant que force d'entrée initiale facilitant l'arrivée de forces de remplacement plus importantes, ou de se déployer en tant que force destinée à montrer la détermination de l'OTAN et sa solidarité en vue d'éviter les crises.

Dans ce cas, la NRF pourrait servir à prévenir un conflit en envoyant une force réduite sur le terrain durant la phase de dissuasion d'une situation en détérioration. L'expérience de l'OTAN en ex-République yougoslave de Macédoine, entre août 2001 et mars 2003, illustre bien le potentiel d'une approche proactive. En effet, l'opération Essentiel Harvest ${ }^{65}$, conduite par

65. Les 19 pays membres de l'OTAN ont lancé la première opération suite à la signature, le 13 août 2001, de l'accord de paix en Macédoine avec l'Albanie. "L'OTAN autorise le déploiement du Quartier général de la 
une force multinationale composée de 3500 hommes était chargée de collecter et de détruire les armes des rebelles albanophones en Macédoine. Sous commandement britannique, elle était composée de quatre bataillons : britanno - néerlandais, grec, italo-turc et franco-germano-espagnol. L'opération s'est achevée le 26 septembre 200166. Elle a été relayée par l'opération Amber Fox ${ }^{67}$, destinée à protéger des observateurs civils internationaux en Macédoine et à superviser la mise en ouvre du plan de paix dans l'ex-République yougoslave de Macédoine. Placée sous commandement allemand et composée d'environ 1000 hommes, la force multinationale a achevé l'opération le 15 décembre 2002. L'OTAN a par la suite, mis sur pied une nouvelle mission le 16 décembre 2002, baptisée Allied Harmony68. Cette dernière regroupait des tâches opérationnelles de soutien aux observateurs internationaux de l'Organisation pour la sécurité et la coopération en Europe (OSCE) et de l'Union européenne, et prenait également en compte la coopération avec l'armée macédonienne, en aidant aux réformes militaires dans le cadre du Partenariat la paix. La mission s'est achevée le 31 mars 2003 et a été relayée par l'opération Concordia, prise en charge par l'Union européenne69. Ces missions ont facilité, en grande partie, une résolution pacifique de la situation et empêché l'escalade de la crise.

La mission de défense collective est celle qui distingue le cadre d'emploi des deux forces, l'Union européenne n'ayant pas de responsabilités dans ce domaine. Pour toutes les autres situations de crise, les missions seront les mêmes. Le choix des États d'intervenir, soit dans le cadre de l'UE, soit dans celui de l'OTAN

\footnotetext{
Force spéciale Harvest" (11 mars 2007), en ligne: OTAN <http://www.nato.int>.

66. "Operation Amber Fox" (11 mars 2007), en ligne: OTAN <http://www.nato.int>.

67. "L'OTAN prolonge l'opération AMBER FOX dans l'ex-République yougoslave de Macédoine" (11 mars 2007), en ligne: OTAN <http://www.nato.int>.

68. "L'OTAN continue de soutenir l'ex-République yougoslave de Macedoine» (11 mars 2007), en ligne : OTAN <http://www.nato.int>.

69. "Council decision" (11 mars 2007), en ligne: Opération de paix $<$ http://www.operationspaix.net/IMG/pdf/DecisionUE_Concordia_18ma rs03_.pdf $>$.
} 
pour faire face à une situation donnée sera toujours politique. Les Européens auront le choix entre trois solutions: tout d'abord, dans le cadre de l'OTAN sans aucune autonomie, ensuite dans un cadre européen avec accès aux moyens de l'OTAN, au travers des accords "Berlin Plus»70, enfin de façon autonome sous la direction d'un pays chef de file ou d'un $\mathrm{GG}$ européen ${ }^{71}$.

Une nouvelle solution doit se mettre en place avec la mise sur pied d'une cellule civilo-militaire au sein de l'EMUE (Étatmajor de l'Union européenne), décidée lors du Conseil européen de Bruxelles des 11 et 12 décembre $2003^{72}$, afin de renforcer la capacité de l'UE à assurer l'alerte rapide, l'analyse des situations, la planification stratégique et la coordination des instruments civils et militaires au cours d'une opération. Un centre d'opérations non permanent sera activé sous certaines conditions pour planifier et conduire une opération de gestion de crise de l'Union, mais seulement dans les cas où les deux premières solutions ne seraient pas valides ou disponibles ${ }^{73}$. Ainsi, le rôle spécifique de l'OTAN sera préservé. Ce souci de ne pas être en

70. Les accords "Berlin Plus", tel qu'il a été précisé, sont fondés sur les quatre principes suivants: la garantie de l'accès à la planification opérationnelle de l'OTAN; la mise à disposition pour l'UE de capacités et de moyens communs de l'OTAN; l'adaptation du système de planification de la défense de l'OTAN d'une manière qui intègre la disponibilité des forces pour des opérations dirigées par l'UE; des options de commandement européen de l'OTAN pour des opérations dirigées par l'UE (rôle du SACEUR adjoint). Ce dernier principe est très important, car il permet de mettre à la disposition de l'UE la direction d'opérations. Cela a été le cas dans le cadre de l'opération Concordia en ARYM puis de l'opération Althéa en Bosnie.

71. Dans le cas où l'UE n'a pas recours aux moyens de l'OTAN, elle désigne une nation cadre qui fournit un état-major national de commandement de l'opération mais qui doit être multinationalisé au moment de la crise, ce qui rend les délais pour une réaction rapide, trop longs. Cela a été le cas de l'opération Artémis en République démocratique du Congo.

72. "Conclusions de la Présidence du Conseil européen" (11 mars 2007), en ligne : Conseil de l'Union européenne <http://www.consilium.europa.eu/ ueDocs/cms_Data/docs/pressData/ fr/ec/79652.pdf $>$.

73. Depuis le 1er janvier 2007, il peut être activé. "Coopération UE-OTAN" (11 mars 2007), en ligne: Ministère des Affaires étrangères <http://www.auswaertigesamt.de/diplo/fr/Europa/Aussenpolitik/ESVP /ZusammenarbeitEU-NATO.html>. 
concurrence directe avec l'Alliance atlantique a d'ailleurs conduit à la création d'une cellule de l'Union européenne au sein de SHAPE (Grand Quartier général des puissances alliées en Europe) et à des arrangements de liaison entre SHAPE et l'EMUE (Étatmajor de l'Union européenne). C'est par cette cellule que l'opération Concordia a été dirigée en ARYM (Ancienne République Yougoslave de Macédoine), d'avril à décembre $2003^{74}$.

Le choix du cadre de l'OTAN ou de l'UE dépendra le plus souvent de la décision des États-Unis d'intervenir aussi ou non, par le biais de l'OTAN, dans une mission de gestion de crises. Il est en effet probable que, dans une situation de crise, si les ÉtatsUnis et les Européens veulent intervenir de façon multilatérale, ils le fassent dans le cadre de l'OTAN, qui possède des forces mieux entraînées et habituées à travailler ensemble. Toutefois, comme les États-Unis n'affectent pas de troupes à la NRF, cette nouvelle structure leur permet de garder le contrôle de l'OTAN sans être impliqués dans des missions qu'ils n'auraient pas choisies. Dans le cas de la survenance d'une crise dans laquelle l'OTAN et l'UE pourraient intervenir, le choix dépendra, dans une large mesure, des instruments qu'il faudra mobiliser. L'UE est à même, en théorie, de répondre à des situations de crise en couvrant tous les aspects d'une situation de conflit, depuis l'imposition de la paix jusqu'à la reconstruction, mettant ainsi en œuvre des moyens militaires et civils. En évitant la concurrence et en favorisant les consultations et la coopération entre l'OTAN et l'UE, les deux organisations doivent s'adapter dans la complémentarité, afin de

74. Les arrangements "Berlin Plus" sont mis en pratique dans le cadre de l'Opération Concordia, le premier déploiement militaire de l'Union européenne dans l'ex-République yougoslave de Macédoine. L'amiral Rainer Feist, adjoint au SACEUR, exerce le commandement de l'opération et des officiers de liaison de l'UE travaillent aux côtés de leurs collègues de l'OTAN dans la structure de commandement de l'OTAN, aussi bien au niveau stratégique, dans une cellule de l'UE au SHAPE à Mons (Belgique), qu'au niveau régional à l'AFSOUTH (Forces alliées du Sud Europe) à Naples (Italie). Sur le terrain, à Skopje, le commandant de la Force - un général de l'armée de terre française - et son état-major, collaborent étroitement avec les hauts représentants militaires et civils de l'OTAN. 
mettre à profit les compétences spécifiques de l'une et de l'autre pour prévenir, gérer et régler crises et conflits.

Cependant, exception faite de l'opération conduite par l'Union européenne en Bosnie-Herzégovine, il n'existe pas vraiment de dialogue politique entre les instances communautaires et alliées ${ }^{75}$. L'opération en Bosnie est un exemple de collaboration entre l'UE et l'OTAN puisque cette opération militaire de l'UE baptisée Althea ${ }^{76}$ est une opération de type Berlin Plus. Ainsi, le 2 décembre 2004 a marqué la fin de l'existence de la Stabilization Force en Bosnie Herzégovine (SFOR), déployée en vertu de l'annexe 11 des accords de Dayton-Paris de 1995. Dans la continuité de la SFOR et en application des décisions prises lors du sommet d'Istanbul, l'Union européenne a repris, dans le cadre de l'EUFOR (European Union Force), les missions qui étaient jusqu'alors menées par l'OTAN en Bosnie-Herzégovine ${ }^{77}$. Dès le 12

75. Des réunions mensuelles, exclusivement consacrées aux opérations en cours en Bosnie-Herzégovine, entre le Conseil de l'Atlantique Nord (CAN) et le Comité politique et de sécurité (COPS), sont l'occasion de discussions autour des questions liées à la sécurité des Balkans pour la mise en œuvre des accords "Berlin Plus". L'OTAN et l'UE avaient mis en place des mécanismes d'échanges réguliers à différents niveaux (ministres des Affaires étrangères de l'OTAN et de l'UE, ambassadeurs de l'OTAN et de l'UE, comités militaires de l'OTAN et de l'UE). Cette organisation a été remise en cause avec l'élargissement de l'UE à 25 nations, les États membres de l'OTAN n'étant pas parvenus à un accord sur les questions pour lesquelles il pourrait y avoir échanges de documents confidentiels. KOENDERS (Bert), "L'évolution du rôle de l'OTAN dans les opérations d'après-conflit", Rapport de la Commission politique de l'assemblée parlementaire de l'OTAN (040 PC 05 F), 3 mai 2005, www.nato.int/docu/pr/2005/p05-076f.html.

76. Il est de tradition de donner un nom de code aux opérations et exercices militaires. Althea est, dans la mythologie grecque, une déesse qui est considérée comme la mère de toutes les mères. Elle est décrite comme étant la femme qui a donné la vie au premier homme et à la première femme. Elle est la déesse de la natalité, elle veille sur toutes les créatures vivantes. Son nom est généralement lié à ses pouvoirs de guérison. Avec ce nom, l'EUFOR veut porter le message de la déesse grecque : sécurité, avenir et bien-être pour tous.

77. L'OTAN est présente en Bosnie depuis la fin de la guerre civile, en décembre 1995, en exécution des accords de paix de Dayton. Elle avait initialement pour tâche de maintenir la paix entre les belligérants de 
juillet 2004, le Conseil de l'Union européenne a adopté une action commune "concernant l'opération militaire de l'Union européenne en Bosnie-et-Herzégovine", l'opération Althea, commandée par le major général britannique Leaky. Elle se compose d'environ 7000 militaires, répartis entre un état-major et trois "Task Forces" d'environ 1800 militaires, ce qui forme la plus vaste opération que l'Union Européenne n'ait jamais mise sur pied. De plus, 2830 militaires allemands, italiens et britanniques sont dédiés au titre de la réserve opérationnelle à partir de leur territoire. L'effectif de cette force européenne correspond à celui déployé par l'OTAN dans le cadre de la SFOR et regroupe à peu près les mêmes unités militaires que celles des pays européens participants déjà présents dans le cadre de l'opération de l'OTAN en Bosnie-Herzégovine. L'opération Althea doit mettre en place des opérations visant la sécurisation du pays en prévenant la résurgence d'actes de violence, ce qui inclut la lutte contre la contrebande, la criminalité et la corruption, facteurs permanents d'instabilité79. L'EUFOR (Force de l'Union européenne) complète et

l'époque, à savoir les Serbes, les musulmans et les Croates. Mais l'OTAN a également aidé à la reconstruction du pays, à la recherche et à l'arrestation des criminels de guerre. En laissant place à une force sous commandement de l'UE, l'OTAN ne disparaît pas complètement de Bosnie puisqu'il reste un état-major à Sarajevo, qui est chargé de la reconstruction d'une armée bosniaque. "The Stabilisation Force (SFOR) in Bosnia and Herzegovina" (11 mars 2007), en ligne: OTAN <http://www.nato.int/issues/sfor/index.html>.

78. Action commune 2004/570/PESC du Conseil du 12 juillet 2004 concernant l'opération militaire de l'Union européenne en Bosnie-etHerzégovine; Journal officiel de l'Union européenne, 28 juillet 2004, L252/ 10.

79. Les missions militaires assignées à Althea sont au nombre de 8 et reprennent l'essentiel des tâches dévolues à la SFOR. D'une part, il y a des missions principales au nombre de 4. Il s'agit en premier lieu, d'assurer une présence militaire afin de dissuader les éléments récidivistes des forces armées bosniaques et d'autres groupes armés; de surveiller l'application des aspects militaires de l'Accord-cadre général de paix et de s'assurer qu'ils continuent d'être respectés afin de prévenir une reprise de la violence. En deuxième lieu, l'EUFOR doit contribuer à un environnement sûr et sécurisé, soutenir le Plan de mise en œuvre de la mission entreprise par le bureau du haut représentant pour la PESC et prévenir les tentatives de renversement du processus de paix. Troisièmement, il s'agit d'exécuter des opérations d'informations à l'appui 
soutient l'action politique et économique de l'Union, le tout coordonné sur place par le représentant spécial de l'UE ${ }^{80}$. L'OTAN maintient une présence dans le pays, avec pour mission de poursuivre des objectifs sécuritaires et militaires liés à la réforme des forces armées bosniaques, à la lutte antiterroriste internationale et à la poursuite des criminels de guerre recherchés par le TPIY. Cette présence est assurée en majorité par des officiers et des soldats américains. Cependant, la décision d'installer le quartier général opérationnel d'Althea au SHAPE (Mons, en Belgique) et de faire de l'adjoint au Commandant suprême des forces alliées en Europe (Deputy SACEUR, en anglais) le commandant de l'opération, montre la volonté de l'UE de gérer l'opération de façon intereuropéenne et autonome par rapport aux États-Unis. Toutefois, à Sarajevo, les deux quartiers généraux EUFOR et OTAN, partagent les mêmes installations au camp Butmir81.

des objectifs politiques de l'UE. Enfin, l'EUFOR doit s'occuper de tous les aspects de l'Accord-cadre qui resteraient à traiter, notamment la gestion de l'espace aérien, les conseils en matière de déminage et d'élimination des munitions et le programme de collecte des armes. D'autre part, il y a aussi des missions secondaires, elles aussi au nombre de 4 . Il s'agit en premier lieu de fournir un soutien aux missions de base prévues par le Plan de mise en œuvre du bureau du haut représentant, ainsi qu'aux autres organismes civils compétents en ce qui concerne la lutte contre le terrorisme et le crime organisé, le retour des personnes déplacées et des réfugiés, l'État de droit et l'application des autres aspects civils de l'Accord-cadre. En deuxième lieu, la force européenne doit apporter une assistance à la réalisation des réformes de défense et donner aux autorités bosniaques, en cas de besoin, des avis militaires et techniques dans le domaine de la sécurité. Troisièmement, il faut soutenir le Tribunal pénal international pour l'ex-Yougoslavie (TPIY). Enfin, il s'agit de fournir un soutien pour l'évacuation des représentants de la communauté internationale, dans la limite des moyens et des capacités. Ibid.

80. Le contrôle politique et les orientations stratégiques concernant l'EUFOR sont de la responsabilité du Comité politique et de sécurité de l'UE (COPS) qui fait rapport au Conseil de l'Union européenne (en formation "Affaires générales et relations extérieures») assisté par le haut représentant pour la PESC.

81. "Nous nous félicitons de la réussite de la transition sans heurt, de la mission de la SFOR à l'opération Althea de l'Union européenne en 
La prise en charge de l'opération Althéa par l'EUFOR n'a donc pas conduit à de véritables changements. L'OTAN et l'EUFOR partagent de nombreuses compétences. Les forces sur le terrain n'ont pas connu de bouleversements qualitatifs ou quantitatifs significatifs puisqu'elles sont, dans leur grande majorité, issues des États européens de l'OTAN et/ou de l'Union européenne et des pays partenaires et candidats à l'adhésion à ces deux institutions. Dans les faits, il ne s'agit que de la relève d'une force européenne déployée dans le cadre euro-atlantique par une autre force européenne relevant de l'Union européenne.

Le remplacement du dispositif de l'OTAN en BosnieHerzégovine par celui de l'Union européenne a toutefois permis de libérer quelques ressources de l'Alliance qui pourront être réorientées au profit d'autres priorités et faciliter les réformes en cours. Il convient donc de souligner l'importance, pour les deux organisations, de se soutenir mutuellement en fonction de l'évolution des événements. Ainsi, on peut imaginer que si la mission de Petersberg (opération Concordia) en Macédoine rencontrait des difficultés sérieuses, l'UE pourrait sans doute demander à l'OTAN de fournir un soutien. Toutefois, d'un point de vue politique, une telle demande mettrait en lumière les limites de l'autonomie de l'action militaire européenne et aurait des conséquences négatives pour le développement de la politique européenne de sécurité et de défense (PESD) ${ }^{82}$.

Bosnie-Herzégovine, dans le cadre des arrangements "Berlin Plus», ainsi que de la coopération étroite qui est maintenue entre nos quartiers généraux respectifs dans ce pays. Cela confirme la valeur d'une coopération étroite entre l'OTAN et l'UE de façon plus générale. Dans le cadre agréé du partenariat stratégique OTAN/UE, nous ferons en sorte de développer encore notre coopération". "Communiqué final Réunion du Conseil de l'Atlantique Nord en session des ministres de la Défense" (11 mars 2007), en ligne : OTAN <http://www.nato.int/docu/pr/2005/p05076f.htm>.

82. La politique extérieure et de sécurité commune (PESC) a été instituée en 1992 par le Traité de Maastricht. La politique européenne de sécurité et de défense (PESD), qui s'inscrit dans la PESC, s'est surtout développée à partir de 1999. L'UE voulait être capable de faire face aux crises. La PESD permet à l'Union européenne de réaliser toutes sortes de missions 
L'OTAN :

vers une conception élargie $\quad$ (2007) 37 R.D.U.S. de la sécurité

Cependant, le cadre d'emploi de ces deux forces n'est pas identique puisque, même si elles comportent certaines tâches opérationnelles communes, le rôle primordial de l'EUFOR reste la stabilisation. La principale différence entre les deux forces va résider dans la chaîne de commandement mise en place dans le cadre des opérations.

\section{La nouvelle structure de commandement militaire}

La NRF est dirigée par la nouvelle structure de commandement de l'Alliance. Cette structure, qui comprend deux commandements stratégiques dont l'un est opérationnel et l'autre fonctionnel, est plus légère, plus efficace et plus facile à déployer que les structures existantes. C'est l'autre décision importante du sommet de Prague qui a consisté en la rationalisation de la structure de commandement de l'OTAN, afin d'offrir "une structure de commandement allégée, plus efficiente, efficace et déployable, en vue de satisfaire les exigences opérationnelles liées à l'éventail complet des missions de l'OTAN" ${ }^{83}$. Lors de leur réunion du 12 juin 2003, les ministres de la Défense de l'Alliance ont approuvé cette nouvelle structure de commandement militaire rationalisée afin de créer une structure de commandement plus souple ${ }^{84}$.

civiles et militaires, allant des interventions humanitaires et de sauvetage à des opérations militaires de maîtrise des crises et de restauration de la paix, en passant par des actions de maintien de la paix. À cet effet, l'Union européenne a décidé de constituer une force comportant jusqu'à 60000 hommes déployables en 60 jours et dont les missions peuvent s'étaler sur une année au moins. L'UE peut aussi recourir si nécessaire, à des capacités humaines et logistiques de l'OTAN pour des missions de la PESD. Le but de l'UE n'est pas de concurrencer l'OTAN mais plutôt de développer une capacité autonome ou complémentaire.

83. "Sommet de Prague 21-22 novembre 2002" (11 mars 2007), en ligne : OTAN <http://www.nato.int>.

84. Auparavant, la structure de commandement militaire reposait sur une division géographique : une pour l'Europe (Commandement allié pour l'Europe SACEUR), une pour l'Atlantique (Commandement allié pour l'Atlantique SACLANT), 7 quartiers généraux étaient subordonnés à ces commandements. 
L'évolution géopolitique ne justifiant plus le maintien de deux zones stratégiques en Europe, la nouvelle structure se compose dorénavant d'un Commandement allié "opérations" (ACO - Allied Command Operations), basé au SHAPE (grand Quartier général des puissances alliées en Europe), à Mons, en Belgique et d'un Commandement allié Transformation (ACT - Allied Command Transformation), basé à Norfolk aux États-Unis. Celui-ci joue un rôle clé dans le développement et la mise en œuvre des capacités réseau-centrées de l'OTAN85 et auprès des États membres. Le premier a toutes les responsabilités opérationnelles ${ }^{86}$ alors que le second supervise la transformation des capacités militaires de l'OTAN en respectant les quatre priorités suivantes : préparer, appuyer et maintenir les opérations de l'Alliance; faire intervenir la Force de réaction de l'OTAN et d'autres capacités projetables; réaliser la pleine capacité opérationnelle ACT; aider à la transformation des capacités des partenaires ${ }^{87}$. L'ACT ${ }^{88}$ définit de nouvelles approches en matière de planification et de constitution de forces et devrait être le fondement même de l'interopérabilité militaire au sein de l'Europe et de part et d'autre de l'Atlantique. Il doit permettre d'améliorer l'entraînement et les capacités, d'élaborer et de mettre à l'épreuve des doctrines et de mener des expériences pour évaluer de nouveaux concepts. Il doit faciliter la diffusion et l'introduction de ces derniers et favoriser l'interopérabilité89.

85. Ces capacités doivent permettre de maîtriser de nouvelles tâches de renseignement, de reconnaissance et de surveillance, la technologie des capteurs ainsi que les systèmes de commandement et de communication. "Defence et security systems" (11 mars 2007), en ligne: EADS <http://www.eads.com/1024/fr/businet/defence/defence.html>.

86. Le SACEUR continuera d'exercer une double fonction, étant aussi commandant du Commandement des forces des États-Unis en Europe.

87. Michel Maisonneuve, "L'institutionnalisation du changement au sein de l'OTAN" (11 mars 2007), en ligne: Revue militaire canadienne <http://www.journal.dnd.ca/frgraph/Vol4/no4/nato_f.asp>.

88. L'ACT exercera une double fonction, étant aussi commandant du Commandement des forces interarmées des États-Unis.

89. Selon le général Lance L. Smith, Commandant suprême allié Transformation, "La chose la plus importante, surtout lorsque le 
$\mathrm{Au}$ niveau stratégique, il n'y a donc plus qu'un commandement assumant des responsabilités opérationnelles, le Commandement allié "opérations" (ACO), commandé par le SACEUR (Commandant suprême des forces alliées en Europe). On passe donc des deux commandements stratégiques opérationnels à un seul90. Le niveau opérationnel se compose de deux commandements de forces interarmées permanents - l'un à Brunssum aux Pays-Bas, l'autre à Naples en Italie et d'un QG interarmées permanent, à Lisbonne au Portugal, plus limité, auquel il sera fait appel pour obtenir une capacité de $\mathrm{GG}$ de groupe de forces multinationales interarmées déployable, basé en mer.

Les résultats qui se dégagent déjà de ces changements sont notables. Les lignes hiérarchiques qui se chevauchaient et semaient la confusion appartiennent au passé, puisque toutes les

processus concerne vingt-six pays, c'est de veiller à ce que quoiqu'on fasse puisse être fait ensemble. En d'autres termes, les forces doivent offrir une interopérabilité». "Interview général Lance L. Smith, Commandant suprême allié Transformation" (11 mars 2007), en ligne : OTAN http://www.nato.int/docu/review/2006/issue3/french/interview. htm>. L'ACT comprend le Centre de guerre interarmées (Norvège), un nouveau centre d'entraînement de forces interarmées (Pologne) et le Centre interarmées d'analyse des enseignements tirés (Portugal). Un élément d'état-major ACT s'occupera en Belgique, principalement des questions relatives aux ressources et à la planification de la Défense. "Briefing : La structure de commandement de l'OTAN" (11 mars 2007), en ligne : OTAN <http://www.nato.int/docu/briefing/nms/html_fr/ nms03.html>

90. Les 13 commandements subordonnés opérationnels passent à 6 seulement. Ces 6 commandements de composante de forces interarmées apportent aux éléments de niveau opérationnel les compétences propres aux différentes armées - terre, mer ou air. Ils sont rattachés, soit au commandement de forces interarmées de Brunssum (composante air à Ramstein en Allemagne, un commandement de composante mer à Northwood au Royaume Uni et un commandement de composante terre à Heidelberg en Allemagne), soit au commandement de forces interarmées de Naples (commandement de composante air à Izmir en Turquie, un commandement de composante mer à Naples et un commandement de composante terre à Madrid en Espagne). "Nouvelle Structure de Commandement de l'OTAN» (11 mars 2007), en ligne: OTAN $<$ http://www.nato.int>. 
opérations relèvent à présent de l'ACO. Une répartition claire des tâches a été établie entre l'ACO et l'ACT nouvellement formé : l'ACO définit les normes que les unités devront satisfaire pour servir dans un commandement de l'OTAN, tandis que l'ACT élabore l'entraînement nécessaire à ces unités. Ainsi, l'ACT, commandé par le SACLANT, n'a plus de responsabilité géographique et n'est plus qu'un état-major fonctionnel, chargé de la transformation alors que l'ACO, commandé par le SACEUR, a une responsabilité exclusivement opérationnelle, couvrant la totalité de l'aire géographique de l'ensemble de l'Alliance. En confiant toutes les responsabilités opérationnelles à un seul commandement et en concentrant le commandement stratégique secondaire sur la transformation en cours et l'amélioration de l'interopérabilité des pays membres, l'OTAN continue sa transformation afin de faire face aux nouveaux défis sécuritaires et aux nouvelles menaces.

Afin de pérenniser ces nouvelles transformations, le sommet d'Istanbul des 28 et 29 juin 200491, a réaffirmé l'importance du rôle mondial de l'Alliance et adopté des mesures pour poursuivre l'amélioration de ses capacités. Le sommet d'Istanbul a souligné que l'OTAN doit projeter la stabilité de trois façons ${ }^{92}$. Tout d'abord, elle doit renforcer ses relations avec ses partenaires. Ensuite, elle doit conduire des opérations dans les Balkans, en Afghanistan et en Méditerranée. Enfin, l'Organisation doit moderniser ses forces armées afin qu'elles puissent mener des missions nouvelles. Ce sommet est un jalon important dans l'histoire de l'OTAN, puisqu'il a permis aux dirigeants des 26 pays

91. "Sommet d'Istanbul; Au sujet du Sommet" (11 mars 2007), en ligne: OTAN <http://www.nato.int>.

92. Comme l'a déclaré le secrétaire général Jaap de Hoop Scheffer à la veille du sommet d'Istanbul : "Notre sommet d'Istanbul fera comprendre que l'OTAN relève les nouveaux défis du XXI ${ }^{e}$ siècle. Il fera comprendre que la nouvelle OTAN, l'OTAN à 26, est une alliance qui agit. Elle agit dans les Balkans et en Afghanistan où nos troupes font, par leur présence, la différence entre la guerre et la paix. Elle agit en Méditerranée, où nos navires conduisent des opérations antiterroristes». "Sommet d'Istanbul; A l'ordre du jour; Opérations" (11 mars 2007), en ligne: OTAN <http://www.nato.int>. 
alliés de se rassembler pour la première fois après le cinquième cycle d'élargissement de l'Alliance, qui a également été le plus important.

La transformation entreprise lors du sommet de Prague des 21 et 22 novembre 2002 afin de mettre en route un processus de modernisation, doit permettre à l'OTAN d'assumer de nouveaux rôles et de nouvelles missions dans de nouvelles régions du monde. À Madrid, les 3 et 4 juin 200393, les ministres des Affaires étrangères de l'OTAN ont déclaré que les nouvelles missions entreprises par l'Alliance en Afghanistan et à l'appui de la Pologne en Irak montraient que la transformation de l'OTAN était bien entamée.

\section{Les opérations de 1'OTAN 94}

L'OTAN doit s'adapter au nouvel environnement stratégique, notamment en acceptant des engagements d'une part, envers de nouveaux objectifs comme la lutte contre le terrorisme et d'autre part, dans de nouveaux théâtres géographiques comme en Afghanistan, en Irak et au Darfour, malgré une transformation en cours de réalisation.

Le 12 septembre $2001^{95}$, les Alliés ont invoqué l'article $5 \mathrm{du}$ Traité de Washington, c'est-à-dire la clause de défense collective. Le Conseil de l'Atlantique Nord a décidé que, s'il était établi que

93. "Communiqué final Réunion ministérielle du Conseil de l'Atlantique Nord Tenue à Madrid le 3 juin 2003" (11 mars 2007), en ligne: OTAN <http://www.nato.int/docu/pr/2003/p03-059f.htm>.

94. Nous n'aborderons pas ici toutes les opérations en cours de l'OTAN mais simplement celles qui témoignent de l'évolution du rôle de l'Organisation. Nous tenons à souligner que l'OTAN intervient aussi en Bosnie, où elle a conservé un quartier général à Sarajevo et en ex-République yougoslave de Macédoine avec un quartier général à Skopje. Enfin, l'OTAN est toujours présente au Kosovo où la KFOR déploya 16800 hommes en 2006 (contre 50000 au départ). Le fonctionnement de cette force a été révisé et chaque brigade est devenue une Task Force, apportant souplesse et mobilité à la mission.

95. "Déclaration du Conseil de L'Atlantique Nord" (11 mars 2007), en ligne : OTAN <http://www.nato.int/docu/pr/2001/p01-124f.htm>. 
l'attaque avait été dirigée depuis l'étranger contre les États-Unis, elle serait assimilée à une action relevant de l'article 5 en vertu duquel une attaque armée contre un ou plusieurs pays membres de l'OTAN est considérée comme une attaque dirigée contre tous. Suite à l'exposé de M. Frank Taylor, ambassadeur extraordinaire des États-Unis et coordonnateur pour le contre-terrorisme, le 2 octobre 2001 devant le Conseil de l'Atlantique Nord, sur les résultats de l'enquête relative aux attentats du 11 Septembre, celui-ci a déterminé que les attaques avaient été dirigées depuis l'étranger et qu'elles seraient donc considérées comme relevant de l'article $5 \mathrm{du}$ Traité de Washington.

Le 4 octobre 200196, l'OTAN a approuvé une série de mesures visant à soutenir les États-Unis. Celles-ci concernaient notamment le domaine du renseignement, le renforcement de la sécurité des installations des États-Unis et des autres Alliés sur leur territoire respectif, les moyens aériens et navals. Ainsi, le Conseil de l'Atlantique Nord a affirmé que l'Alliance était prête à déployer des éléments de ses forces navales permanentes en Méditerranée orientale pour assurer une présence de l'OTAN. Peu après, l'OTAN a lancé sa toute première opération antiterroriste : Eagle Assist. Á la demande des États-Unis, de la mi-octobre 2001 à la mi-mai 2002, 7 appareils AWACS de l'OTAN ont été envoyés pour aider à patrouiller l'espace aérien au-dessus des États-Unis. C'était la première fois que les moyens militaires de l'OTAN étaient déployés à l'appui d'opérations relevant de l'article 5.

Le 26 octobre 2001, l'Alliance a lancé sa seconde opération antiterroriste, Active Endeavour. Cette opération s'inscrit dans le droit fil de la réaction immédiate de l'OTAN aux attentats terroristes perpétrés le 11 Septembre 2001 contre les États-Unis. Dans le cadre de cette opération, des navires de l'OTAN effectuent des missions de patrouille en Méditerranée. Ils surveillent la

96. "Déclaration à la presse du Secrétaire général sur la décision du Conseil de l'Atlantique Nord concernant l'application de l'article 5 du Traité de Washington en conséquence des attaques perpétrées le 11 septembre contre les Etats-Unis" (11 mars 2007), en ligne: OTAN $<$ http://www.nato.int $>$. 
navigation et escortent dans la traversée du détroit de Gibraltar des navires non militaires. Il s'agit de "surveiller le trafic maritime (...) afin de contribuer à détecter et à décourager les activités terroristes»97. L'opération vise un double but: démontrer la solidarité de l'OTAN et sa résolution dans la lutte contre le terrorisme, ainsi que contribuer à détecter et à décourager les activités terroristes en Méditerranée. Le 10 mars 2003, l'opération a été élargie à l'escorte de navires civils toujours dans le détroit de Gibraltar.

Lors de la réunion des ministres des Affaires étrangères à Reykjavik, le 15 mai $2002^{98}$, lord Robertson a déclaré que le cadre dans lequel s'inscrirait la transformation de l'OTAN serait : "[la] lutte contre le terrorisme, [l']élargissement, [de] nouvelles capacités et nouvelles relations"99. Par cette déclaration est ainsi affirmée la détermination de l'Alliance à conduire des opérations partout où cela sera nécessaire pour combattre le terrorisme. Cette déclaration est historique, au moins d'un point de vue théorique, parce qu'elle a mis fin au débat sur ce qui est et ce qui n'est pas la zone d'opérations de l'OTAN ${ }^{100}$. En effet, la lutte contre

97. Opération Active Endeavour, supra note 31. Les forces de l'OTAN ont contrôlé plus de 75000 navires et arraisonné une centaine de bâtiments suspects. En outre, plus de 480 navires ont profité d'une escorte de l'OTAN.

98. "Modernisation de l'OTAN; A Reykjavik, les ministres des Affaires étrangères de l'Alliance définissent la marche à suivre" (11 mars 2007), en ligne : OTAN <http://www.nato.int>.

99. Ibid.

100. Cependant, Robert Bell, ancien secrétaire général adjoint de l'OTAN pour le soutien de la Défense, explique que, malgré la clarté des déclarations prises lors des différents sommets de Prague et d'Istanbul, des désaccords existent sur les missions de l'OTAN en général et de la NRF en particulier. Ainsi, le déploiement de la NRF en Afghanistan pour assurer la sécurité des élections locales, a soulevé le problème de l'implication de l'OTAN dans des missions qui ne concernent plus exclusivement la défense collective. L'évocation d'un déploiement de l'OTAN au Liban, suite à une mission mandatée par l'ONU, a de nouveau fait apparaître des divergences quant aux théâtres d'opérations dans lesquels l'OTAN pouvait se déployer. Robert Bell, "Le mythe de Sisyphe et la NRF" (11 mars 2007), en ligne: Revue de l'OTAN <http://www.nato.int/docu/review/ 2006/issue3/french/art4.html>. Il 
le terrorisme sera au cour de toutes les réunions ultérieures et lors du sommet de Prague des 21 et 22 novembre 2002, les chefs d'État et de gouvernement des pays de l'OTAN ont adopté un ensemble de mesures parmi lesquelles un nouveau concept militaire de défense contre le terrorisme et un plan d'action pour un partenariat en matière de terrorisme, ceci dans le but de préparer l'OTAN à relever le défi que pose le terrorisme. À Istanbul, en juin 2004, les membres de l'OTAN ont aussi approuvé un ensemble renforcé de mesures propres à accroître la contribution de l'Alliance à la lutte contre le terrorisme en développant notamment, le partage des données du renseignement par un réexamen des structures actuelles du renseignement à l'OTAN et grâce à l'Unité du renseignement sur la menace terroriste, implantée au siège de l'OTAN.

Les États-Unis ont joué un grand rôle dans l'évolution des tâches de l'OTAN puisqu'ils ont tout mis en œuvre pour que la doctrine de l'OTAN reçoive une nouvelle définition. Touchés en plein cour par les attentats du 11 Septembre 2001, les États-Unis ont voulu faire de la lutte contre le terrorisme leur priorité et celle de l'OTAN. Cependant, cette nouvelle direction peut se heurter à certaines limites. D'une part, la lutte contre le terrorisme passe par un meilleur partage des renseignements. Or, on sait que ce domaine est l'un des plus sensibles au niveau militaire et qu'il est directement lié à l'intégrité nationale des États. D’autre part, en tant qu'organisation purement militaire, l'OTAN n'a pas les moyens nécessaires pour lutter contre le financement du terrorisme, nerf de la guerre. Les États-Unis souhaitent

faut souligner que les divergences sont liées à une différence de points de vue entre, d'un côté, ceux qui veulent défendre une conception "eurocentrique" de l'Alliance et, de l'autre côté, les défenseurs d'une vision "euro-atlantique». Il s'agit là de deux visions différentes du rôle des ÉtatsUnis et de l'Union européenne. Tomas Valasek, "Le choc de la réalité" (11 mars 2007), en ligne: Revue de l'OTAN <http://www.nato.int/docu/ review/2006/issue2/french/art3.html>.

Pour une description des différents points de vue sur le rôle de l'article 5. Sebestyén L. v. Gorka, "L'invocation dans son contexte" (11 mars 2007), en ligne: Revue de l'OTAN <http://www.nato.int/docu/review/2006/ issue2/french/art1.html>. 
néanmoins influer sur la direction vers laquelle doit se tourner l'Organisation, à savoir une organisation de sécurité collective qui ne doit plus avoir de limites géographiques et fonctionnelles d'intervention.

Les États-Unis reconnaissent que l'Alliance a fait beaucoup de progrès depuis la fin de la Guerre froide mais, selon eux, au lendemain du 11 Septembre 2001, elle n'est pas encore prête à mener des opérations de lutte contre le terrorisme. C'est pourquoi, ils ont d'abord préféré réagir en dehors du cadre de l'OTAN en réunissant une coalition ${ }^{101}$ afin de faire tomber le régime taliban. Lors de la Conférence de Bonn de $2001^{102}$ au cours de laquelle les dirigeants de l'opposition afghane se sont réunis pour amorcer le processus de reconstruction de leur pays, l'ISAF (International Security Assistance Force ou FIAS, Force internationale d'assistance à la sécurité) est créée. Le résultat des accords de Bonn fut la formation d'un gouvernement intérimaire, un plan pour la paix et la sécurité, la reconstruction du pays, la réouverture des institutions-clés et la protection des droits de l'homme. Le Conseil de sécurité des Nations Unies avait alors mandaté le déploiement d'une force multinationale ${ }^{103}$. Il faut préciser que l'ISAF n'est pas une force des Nations Unies mais une coalition de pays volontaires, déployée sous l'autorité du Conseil

101. On peut aussi estimer que les États-Unis ne souhaitaient pas être paralysés par la nécessité d'un consensus au sein du Conseil de l'Atlantique Nord si la mission engagée venait à évoluer.

102. Cette conférence s'est déroulée du 27 novembre au 5 décembre 2001 , sous les auspices de l'ONU (UN-Talks on Afghanistan). L'ONU a tenu le rôle de médiateur, l'Allemagne celui de simple hôte. La conférence répondait au "Plan en cinq points" présenté par l'envoyé spécial des Nations Unies, Lakhari Brahimi, le 13 novembre 2001. Julien Thorel, "Les implications de l'Allemagne dans la reconstruction de l'Afghanistan" (2005) 9 géostratégiques 59 aux p. 59-76; "Accord définissant les arrangements provisoires applicables en Afghanistan en attendant le rétablissement d'institutions étatiques permanentes" (11 mars 2007), en ligne: Geopolitis <http://www.geopolitis.net/LES\%20DOCUMENTS/ ACCORD\%20SUR\%20L'AFGHANISTAN.pdf>.

103. La situation en Afghanistan, Rés. CS 1386, Doc. off. CS, 4443e séance, S/RES/1386 (2001); La situation en Afghanistan, Rés. CS 1444, Doc. off. CS, $4651^{\mathrm{e}}$ séance, S/RES/1444 (2002). 
de sécurité des Nations Unies, financée par les pays fournisseurs de troupes.

L'OTAN a pris officiellement le commandement de l'ISAF'104 le 11 août 2003105, succédant ainsi à la Force, dirigée par l'Allemagne, les Pays-Bas et le Canada106 et menant ainsi sa première mission en dehors de la zone euro-atlantique en prenant la relève de la mission des Nations Unies chargée d'assurer la sécurité à Kaboul. Les effectifs de l'ISAF sont de 6300 hommes, venant de 36 pays, membresio7 ou non de l'OTAN 108 et placés sous la direction politique du Conseil de l'Atlantique Nord. Le commandement et le contrôle stratégiques sont exercés par le Grand Quartier général des puissances alliées en Europe (SHAPE), à Mons en Belgique, en application des orientations politiques données par le Conseil de l'Atlantique Nord. Il faut souligner que l'OTAN demeure le commandant en chef de la mission dans son intégralité, même si différents États assurent le commandement du quartier général. Le recours à l'OTAN pour assurer la relève permet de résoudre le problème lié à la nécessité

104. "L'OTAN est prête pour sa mission en Afghanistan" (11 mars 2007), en ligne: OTAN <http://www.nato.int/docu/update/2003/08-august/ f0806a.htm>.

105. L'engagement découle de la décision prise le 16 avril 2003 par le Conseil de l'Atlantique Nord et souligne la volonté à long terme de tous les Alliés en faveur de la stabilité et de la sécurité pour le peuple afghan. "Même nom, même drapeau, même mission : l'OTAN renforce son soutien à l'ISAF" (11 mars 2007), en ligne : OTAN <http://www.nato.int>.

106. L'ISAF a connu plusieurs phases. L'ISAF I a été dirigée par le RoyaumeUni, l'ISAF II par la Turquie, l'ISAF III par l'Allemagne, le Canada et les Pays-Bas et l'ISAF IV par l'OTAN. Diego Ruiz Palmer, "La route de Kaboul" (2003) 2 Revue de l'OTAN 11. L'ISAF $V$ a été dirigée par le Canada, l'ISAF $V I$ est passée sous le commandement de l'Eurocorps, opérant sous les auspices de l'OTAN avec un commandement commun des forces. La Turquie a pris le commandement de la septième mission de l'ISAF. Enfin actuellement, il revient à l'Italie de commander l'ISAF VIII.

107. Les pays membres de l'OTAN contributeurs de troupes sont au nombre de 26. "L'Otan en Afghanistan" (11 mars 2007), en ligne: OTAN <http://www.nato.int>.

108. Dix pays non OTAN fournissent des troupes à l'ISAF. Des pays non membres de l'OTAN peuvent participer à cette force puisqu'il ne s'agit pas d'une opération de l'OTAN mais d'une mission dirigée par l'OTAN. Ibid. 
L'OTAN :

vers une conception élargie $\quad$ (2007) 37 R.D.U.S. de la sécurité

de trouver, tous les six mois, de nouveaux pays pour diriger la mission. La prise en charge du commandement par l'OTAN offre l'avantage d'assurer la pérennité de la direction de la mission. De plus, elle permet de ne pas faire peser sur une nation tout le poids d'une lourde logistique.

Cette mission consistait uniquement, à l'origine, à assurer la sécurité à Kaboul et dans les environs afin que l'Autorité intérimaire afghane et le personnel des Nations Unies soient en mesure d'agir dans un environnement sûr. Le soutien de l'OTAN s'est traduit par la mise en place d'un quartier général sur le théâtre pour le commandement et la coordination de l'opération; la désignation d'un commandant de la force par le Commandant suprême des forces alliées en Europe; la coordination, le commandement et le contrôle stratégiques, exercés par le Grand quartier général des puissances alliées en Europe (SHAPE) et la direction et coordination politiques par le Conseil de l'Atlantique Nord.

Le Conseil de sécurité des Nations Unies109 a élargi le mandat de l'ISAF à l'ensemble de l'Afghanistan, permettant ainsi à l'OTAN d'opérer en dehors de Kaboul en reprenant la responsabilité de l'Équipe de reconstruction provinciale (PRT) dirigée par l'Allemagne. Les PRT sont des petites équipes de civils et de militaires chargées d'assurer la sécurité des travailleurs humanitaires et de contribuer aux travaux de reconstruction. Les dirigeants alliés ont par ailleurs renforcés la présence de l'OTAN dans le pays ${ }^{110}$ par le biais de l'établissement d'Équipes de reconstruction provinciale (PRT) supplémentaires, combinées à des équipes civilo-militaires œuvrant pour étendre l'autorité du gouvernement central afin de faciliter le développement et la reconstruction et de fournir les troupes et les équipements

109. La situation en Afghanistan, Rés. CS 1510, Doc. off. CS, $4840^{e}$ séance, S/RES/ 1510 (2003).

110. "Communiqué final Réunion du Conseil de l'Atlantique Nord en session des ministres de la Défense tenue à Bruxelles le lundi ler décembre 2003" (11 mars 2007), en ligne: OTAN <http://www.nato.int/ docu/pr/2003/p03-148f.htm>. 
nécessaires à cette expansion. En présence du président afghan Hamid Karzai, l'OTAN a annoncé qu'elle assumerait le commandement de quatre nouvelles PRT - les PRT sous direction britannique à Mazar-e-Sharif et Maimana, la PRT sous direction allemande à Feyzabad et la PRT sous direction néerlandaise à Baghlan - en plus de la PRT existante sous direction allemande à Kundu ${ }^{111}$. En plus de ces cinq PRT, l'OTAN a établi une base de soutien logistique près de Mazar-e-Sharif et des présences satellites temporaires à Sar-e-Pol, Samangan et Sherberghan. L'ISAF est ainsi en mesure de contribuer à la sécurité de neuf provinces afghanes, couvrant une zone d'opérations de 3600 kilomètres carrés à et autour de Kaboul et de 185000 kilomètres carrés dans le nord du pays. Ce processus a été achevé le $1^{\text {er }}$ octobre 2004, marquant ainsi la fin de la première phase d'extension du mandat de l'ISAF.

Le 10 février 2005, l'OTAN a annoncé une nouvelle extension de la mission de l'ISAF dans l'ouest de l'Afghanistan. Le 31 mai 2005, deux PRT supplémentaires dans les provinces de Herat et de Farah sont passées sous le commandement de l'ISAF. L'extension dans l'ouest de l'Afghanistan s'est poursuivie au cours de l'été 2005 avec l'opérationnalité de deux autres PRT, l'une à Chagcharan, capitale de la province de Ghor, et la seconde à Qal'eh-Now, capitale de la province de Baghdis. Avec cette extension de la mission de l'ISAF, l'assistance à la sécurité couvrira $50 \%$ du territoire afghan ${ }^{112}$.

Les ministres des Affaires étrangères des pays de l'Alliance ont approuvé, le 8 décembre $2005^{113}$, un plan afin d'étendre la

111. "Communiqué du sommet d'Istanbul publié par les chefs d'État et de gouvernement participant à la réunion du Conseil de l'Atlantique Nord tenue à Istanbul le 28 juin 2004" (11 mars 2007), en ligne : OTAN $<$ http://www.nato.int>.

112. «Briefing : Afghanistan 8. Extension progressive de la présence de l'OTAN en Afghanistan" (11 mars 2007), en ligne : OTAN <http://www.nato.int/ docu/briefing/afghanistan-2006/html_fr/afghanistan08.html >.

113. "Déclaration; Réunion du Conseil OTAN-Russie au niveau des ministres des Affaires étrangères tenue à Bruxelles le jeudi 8 décembre 2005" (11 mars 2007), en ligne : OTAN <http://www.nato.int>. 
mission de l'ISAF vers le sud. Ce plan a été mis en œuvre le 31 juillet 2006 lorsque l'ISAF a pris la relève des forces de la Coalition que dirigeaient jusqu'alors les États-Unis dans la région sud de l'Afghanistan, élargissant ainsi sa zone d'opération à six provinces supplémentaires - Daikondi, Helmand, Kandahar, Nimroze, Uruzgan et Zaboul - et assumant le commandement de quatre équipes de reconstruction provinciales supplémentaires.

Le 5 octobre $2006^{114}$, l'ISAF a pris la relève des forces militaires internationales de la Coalition dans l'est de l'Afghanistan. Cette extension géographique s'est accompagnée d'un accroissement du rôle de l'OTAN, puisque celle-ci doit déployer des équipes d'encadrement et de liaison opérationnelles auprès des unités de l'armée nationale afghane. L'OTAN dirige environ 30000 soldats originaires de 37 pays et 25 équipes de reconstruction provinciales (PRT). Il s'agit de la première et de la plus grande opération terrestre de l'OTAN hors d'Europe.

Les résultats de l'ISAF ont été plutôt satisfaisants lorsque son action était limitée géographiquement et, on a pu noter que des progrès ont été accomplis dans le cadre de l'action menée pour apporter la paix et la stabilité en l'Afghanistan ${ }^{115}$. Cependant, l'extension de la mission de l'ISAF s'est accompagnée d'une détérioration de la situation et le coût en vies humaines n'a cessé d'augmenter. L'engagement de l'OTAN dans ce pays met à l'épreuve sa capacité à relever les défis du nouvel ordre de sécurité. La mission en Afghanistan a été l'occasion pour l'Organisation, d'élargir ses zones d'intervention géographiques et fonctionnelles, en lui permettant de prendre part à d'autres volets que celui strictement militaire, en participant, par exemple, à la lutte antidrogue ou à la formation de la police. Mais le succès n'est pour l'instant pas total et ne valide pas la transformation voulue

114. «Force internationale d'assistance à la sécurité (FIAS); Contribuer à instaurer la sécurité, la stabilité et encourager le développement en Afghanistan" (11 mars 2007), en ligne: OTAN <http:// www.nato.int/issues/isaf/index-fr.html>.

115. "Communiqué final du 9 juin 2005; Réunion du Conseil de l'Atlantique Nord en session des ministres de la Défense" (11 mars 2007), en ligne : OTAN <http://www.nato.int>. 
par les États-Unis de l'OTAN, en une organisation mondiale de la sécurité. L'avenir de la mission de l'ISAF sera décisif pour son évolution car, selon les hauts fonctionnaires de l'Organisation eux-mêmes : "[...] un Afghanistan failli signerait la faillite de l'OTAN"116. L'Afghanistan reste donc la priorité de l'OTAN"17.

Toutefois, d'autres missions sont en cours, comme celle permettant d'illustrer la nouvelle orientation de l'Alliance en Irak. Conformément à la résolution 1546 du Conseil de sécurité de l'ONU118, tous les membres de l'Alliance contribuent à la mission entreprise par l'OTAN pour aider le gouvernement iraquien démocratiquement élu, à former et à équiper les forces de sécurité iraquiennes, afin qu'il soit le plus tôt possible en mesure d'assumer pleinement la responsabilité de la stabilité du pays et de la sécurité de la population. Au sommet d'Istanbul, en juin 2004, les membres de l'OTAN ont décidé d'aider le gouvernement provisoire irakien à entraîner ses forces de sécurité en mettant en place une Mission OTAN qui serait chargée de s'occuper de cet entraînement dans le pays même et dans d'autres endroits de la région, ou encore en Europe119. Le premier contingent affecté à la Mission a été déployé au mois d'août

116. "Déclaration du sommet de Riga" (11 mars 2007), en ligne: OTAN $<$ http://www.nato.int>.

117. Le 26 janvier 2007, plusieurs membres de l'Alliance ont fait part de leur intention d'envoyer des troupes supplémentaires en Afghanistan et de leur volonté de renforcer l'aide et d'augmenter le nombre des personnels civils afin d'intensifier le travail de reconstruction et de développement. "Chronologie FIAS : janvier 2007" (11 mars 2007), en ligne : Opérations de paix <http://www.operationspaix.net/spip.php?page= chronologie $\&$ id_mot $=85 \&$ date $=2007-01>$.

118. La situation entre l'Iraq et le Koweït, Rés. CS 1546, Doc. off. CS, 4987e séance, S/RES/1546 (2004).

119. Certains membres des forces irakiennes suivent un entraînement au Centre de guerre interarmées de l'OTAN de Stavanger, en Norvège, d'autres à l'École de l'OTAN d'Obergammergau, en Allemagne. La France quant à elle, s'est engagée dans une mission bilatérale de formation des policiers irakiens au Gatar. L'Espagne pour sa part, forme des démineurs irakiens dans une base proche de Madrid. Daniel Speckhard, "Contribuer à la stabilisation de l'Irak" (11 mars 2007), en ligne: OTAN <http://www.nato.int>. 
2004120. En septembre 2004, le Conseil de l'Atlantique Nord $(\mathrm{CAN})^{121}$ a étendu le mandat de la Mission à la fondation d'un centre d'entraînement, d'enseignement et de doctrine en Irak, faisant passer l'effectif du contingent initial de 50 à 300 hommes.

De plus, l'OTAN coordonne l'aide matérielle et technique aux autorités irakiennes à travers un groupe de coordination de la formation et de l'équipement, mis en place au siège de Bruxelles en octobre $2004^{122}$. L'OTAN continue en même temps d'apporter un soutien à la Pologne pour la direction de la division multinationale dans le centre sud de l'Irak.

Toutefois, la Mission de formation de l'OTAN en Irak ne reçoit, de la part de certains Alliés, qu'un appui marginal. Cette faible implication peut s'expliquer par la multiplication des engagements et des déploiements en Afghanistan et ailleurs. De plus, les "clauses suspensives nationales" - c'est-à-dire les restrictions imposées aux forces et autres personnels affectés à des missions de l'OTAN - compliquent le déroulement de la mission de formation de l'OTAN en Irak. Ces clauses empêchent par exemple, certains militaires affectés aux états-majors multinationaux de l'OTAN d'y prendre part, ce qui peut nuire au bon déroulement de la mission irakienne et en soi, au principe du consensus. Malgré la réticence de certains membres, l'OTAN a

120. Cette mission est placée sous la conduite du général de division Carel Hilderink (NL) puis du général de division Agner Rokos (DK); ce dernier fera office d'adjoint au commandant de la Mission, le général de corps d'armée David Petraeus (US) qui assure également le commandement du Multinational Security Transition Command in Iraq. "L'OTAN envoie un premier groupe d'officiers en Irak" (11 mars 2007), en ligne : Comité de Surveillance OTAN <http://www.csotan.org>.

121. Le Conseil de l'Atlantique Nord est composé de représentants permanents de tous les pays membres, qui se réunissent au moins une fois par semaine. Le Conseil peut aussi se réunir avec les ministres des Affaires étrangères, les ministres de la Défense ou les chefs d'État et de gouvernement. Le CAN est la plus haute instance de prise de décisions de l'OTAN. OTAN, "Manuel de l'OTAN", supra note 2 à la p. 35.

122. Le Danemark et la Roumanie ont fourni du matériel militaire tandis que la Grèce, la Norvège et le Luxembourg ont consenti une assistance financière. "Premier stage OTAN de formation pour l'Iraq Organisé en Norvège" (11 mars 2007), en ligne : OTAN <http://www.nato.int>. 
prouvé qu'elle pouvait sortir de son champ d'action et continuer d'exister. Dans ce même but, l'Organisation veut instaurer un réseau de relations avec certains pays du Moyen-Orient, en collaborant avec eux dans les domaines de la lutte contre le terrorisme et de l'interdiction des ADM et ainsi qu'en favorisant des opérations de stabilité.

La dernière mission de l'OTAN se déroule au Darfour. C'est l'Union africaine (UA) qui a fait appel à l'OTAN afin de renforcer les moyens de l'AMIS (African Union Mission in Sudan) et sa Force de paix au Darfour123. Son objectif est de faire passer ses effectifs de 2700 soldats à 7 700. Pour cela, les pays africains composant cette mission qui ont les troupes nécessaires mais pas les moyens de les équiper, de les transporter comme de les soutenir, ont demandé à l'OTAN, le 26 avril 2005 par le biais de l'Union africaine ${ }^{124}$, d'examiner les possibilités d'apporter un soutien logistique à l'opération de maintien de la paix, menée par l'Union africaine au Darfour. Le 17 mai 2005125, M. Alpha Oumar Konaré, président de l'Union africaine, est venu en visite à l'OTAN afin de donner des précisions sur le type d'aide dont l'Union avait besoin. Le 25 mai 2005126, le Conseil de l'Atlantique Nord a approuvé une première série de mesures militaires que l'Alliance pourrait prendre pour soutenir la mission de l'Union africaine, notamment dans les domaines suivants : le transport aérien stratégique ; la formation, par exemple sur les plans du commandement, du contrôle et de la planification opérationnelle, ainsi que

123. "L'OTAN apportera un soutien logistique à l'UA au Darfour» (11 mars 2007), en ligne: USINFO <http://usinfo.state.gov/fr/Archive/ 2005/Jun/10-239970.html>.

124. "L'OTAN va étudier la possibilité de soutenir l'Union africaine au Darfour» (11 mars 2007), en ligne : OTAN <http://www.nato.int>.

125. "Le président de l'Union africaine en visite à l'OTAN" (11 mars 2007), en ligne : OTAN <http://www.nato.int>.

126. "Déclaration du porte-parole de l'OTAN sur le soutien à l'Union Africaine pour le Darfour" (11 mars 2007), en ligne : OTAN <http://www.nato.int>. 
l'amélioration de la capacité de la mission d'utiliser le renseignement ${ }^{127}$.

Le 26 mai $2005^{128}$, en Éthiopie, le secrétaire général de l'OTAN, M. Jaap de Hoop Scheffer, a déclaré que l'Alliance était prête à apporter une aide logistique à l'Union africaine pour sa mission au Darfour et à l'assister pour la formation des personnels engagés dans cette mission, accomplissant ainsi pour la première fois de son histoire, une opération sur le continent africain. L'OTAN doit transporter des soldats de la paix appartenant aux pays africains fournisseurs de troupes. Elle formera les troupes de l'Union africaine pour l'administration d'un quartier général multinational et la gestion des données du renseignement. Grâce aux avions gros porteurs canadiens et américains, l'OTAN devrait monopoliser le transport de troupes africaines.

En juillet 2005, l'OTAN a transporté par avion au Darfour plus de 2000 soldats supplémentaires ${ }^{129}$ de l'Union africaine, afin d'aider cette dernière à renforcer sa présence sur place. Une mission de transport aérien de l'OTAN qui s'est déroulée jusqu'en septembre 2005. En plus de cela, l'OTAN a apporté son aide pour former le personnel de l'Union africaine dans les principales fonctions de $\mathrm{BG}$, à savoir : le commandement et le contrôle, la logistique et la planification. La coordination du transport aérien se fait à partir du SHAPE. Une cellule spéciale d'une vingtaine de personnes s'occupant des mouvements aériens de l'Union africaine au siège de cette dernière à Addis-Abeba, coordonne les mouvements des troupes arrivant sur le terrain. L'Union africaine a l'entière direction des opérations.

127. «Feu vert au soutien de l'OTAN à l'Union africaine pour le Darfour» (11 mars 2007), en ligne : OTAN <http://www.nato.int/docu/update/2005/ 06-june/f0609a.htm>.

128. Le président de l'Union africaine en visite à l'OTAN, supra note 125 .

129. Les avions américains $\mathrm{C} 130$ et $\mathrm{C} 17$ ont acheminé environ 1350 soldats dans la région, alors que le Royaume-Uni assurait le transport de 680 autres. "Assistance de l'OTAN à l'Union africaine pour le Darfour" (11 mars 2007), en ligne : OTAN <http://www.nato.int>. 
Dans le cadre de cette opération, le Conseil de l'Atlantique Nord a réaffirmé sa détermination à œuvrer avec l'Union européenne, les Nations Unies et tous les donateurs concernés, dans un esprit de complémentarité et de transparence130. De leur côté, les ministres des Affaires étrangères de l'UE, réunis à Bruxelles doivent se mettre d'accord sur un paquet de mesures à proposer au nom de l'Union. Présente sur place depuis six mois, mais peu visible131, l'Union européenne ne veut pas se faire voler la vedette par l'OTAN, nouvelle venue dans la région. Les Vingtcinq, qui sont les plus gros pourvoyeurs d'aide humanitaire au Darfour (445 millions d'euros depuis 2003), se disent prêts "à apporter tout le soutien possible aux efforts militaires, policiers et civils" de l'Union africaine $^{132}$. Le Conseil de l'Union européenne a soutenu le projet d'une action commune pour appuyer l'Union africaine. Le contrôle politique et la direction stratégique de l'action sont confiés au Comité politique et de sécurité (COPS) et la coordination est dévolue au représentant spécial de l'Union européenne, M. Pekka Haavisto ${ }^{133}$. L'action de l'UE se décompose en deux volets : le premier est civil et doit permettre la mise à disposition de forces de police européennes pour le commandement policier d'AMIS II (African Union Mission in

130. "L'expérience opérationnelle que l'OTAN a acquise avec ses forces armées dans les domaines des activités postérieures à un conflit et de stabilisation, démontre clairement l'importance de poursuivre une coopération étroite entre l'Alliance et les autres organisations internationales, ainsi que les autres acteurs, de même que la nécessité d'optimiser cette coopération". "Communiqué final; Réunion du Conseil de l'Atlantique Nord en session des ministres de la Défense le 9 juin 2005" (11 mars 2007), en ligne : OTAN <http://www.nato.int>.

131. L'UE a contribué en 2004 au déploiement de la commission du cessezle-feu et de la première mission de l'UA au Darfour: contribution financière complétée par la présence d'experts travaillant avec les soldats de l'UA. L'Union européenne a engagé, en novembre 2004, une enveloppe de 80 millions d'euros pour financer la mission de l'Union africaine. "Darfour" (11 mars 2007), en ligne : ministère des Affaires étrangères $<$ http://www.diplomatie.gouv.fr/fr/rubrique.php3?id_rubrique=1091>.

132. "Rôles de l'OTAN et de l'Union européenne au Darfour" (11 mars 2007), en ligne : centre démocrate humaniste <http://www.lecdh.be $>$. .

133. Action commune 2005/556/PESC du Conseil du 18 juillet 2005 portant nomination du représentant spécial de l'UE pour le Soudan; Journal officiel de l'Union européenne, 20 juillet 2005, L188/43. 
Sudan) ; la formation du personnel et la mise en place d'une unité de police. Le second est militaire et doit fournir une assistance à la chaîne de commandement d'AMIS II; mettre à disposition des observateurs militaires; participer à la formation de militaires et aider au transport stratégique et tactique134 ainsi qu'à l'hébergement des soldats de l'UA.

Le partage des tâches entre l'Union européenne et l'OTAN n'est pas très lisible puisque les deux organisations interviennent parfois dans des domaines similaires, comme le transport aérien ou la formation des troupes de l'Union africaine. Concernant le secteur aérien, la bataille avec l'Union européenne se joue entre Américains et Français au niveau de la surveillance ${ }^{135}$, la France disposant d'une importante base militaire au Tchad ${ }^{136}$. Pour l'Union européenne137, celle-ci s'est d'ailleurs engagée à transporter un bataillon complet de soldats sénégalais avec leur matériel ; l'Allemagne a prévu six avions pour le transport de huit bataillons de soldats de l'Union africaine et celui de 380 policiers ; la Grèce a offert l'utilisation de deux avions et le Luxembourg a

134. «Conseil Affaires générales et relations extérieures 10.813/05» (11 mars 2007), en ligne: Conseil de l'Union Européenne

<http://www.consilium.europa.eu/cms3_applications/Applications/new sRoom/loadBook.asp?target=2005\&bid=71 \&lang=2\&cmsId=349>.

135. Les États-Unis disposent de capacités militaires bien plus importantes que l'UE et pourraient par exemple, fournir des avions de surveillance du territoire Awacs. Mais, la décision du président Konaré de solliciter l'OTAN et pas seulement l'UE, correspond au choix politique de vouloir impliquer les États-Unis et d'éviter, comme il l'a répété durant son séjour aux instances de l'UE, tout "partenariat exclusif".

136. La France a présenté à Bruxelles une série de contributions pour appuyer la mission, dont la mise à disposition d'une dizaine d'officiers planificateurs et d'experts en logistique, 1200 heures de vols d'avions gros porteurs, des moyens aériens de surveillance, avec un Mirage F1CR, un Atlantique2 et un ravitailleur Boeing C135. La France a mis des moyens militaires à la disposition de l'UE mais aucun pour l'OTAN. "Activité : La coopération militaire entre les forces françaises et américaines" (11 mars 2007), en ligne : Ambassade de France aux EtatsUnis <http://www.ambafrance-us.org/fr/ambassade/defense/defense3. asp>.

137. "Activité de l'Union européenne; Politique étrangère et sécurité" (11 mars 2007), en ligne : Europa <http://europa.eu>. 
offert une enveloppe de 75000 euros pour les frais découlant de l'utilisation d'avions nolisés. L'OTAN a, quant à elle, transporté environ 3800 soldats appartenant aux pays africains fournisseurs de troupes, dont elle assure la coordination à partir de l'Europe. Le chevauchement de l'action des deux organisations concerne aussi la formation des soldats, puisque chacune d'elles s'y est engagée. La formation est basée sur la planification et l'étude de foyers stratégiques, grâce à des techniques permettant d'aboutir à une analyse globale, identifiant les moyens de sortie de crises. De plus, les officiers de l'UA se sont montrés particulièrement intéressés par l'expérience de l'OTAN en Afghanistan et par le concept de $\mathrm{NRF}^{138}$. L'organisation entre les différents intervenants est d'autant plus compliquée que c'est l'Union africaine qui pilote les opérations depuis son siège à Addis-Abeba.

La mission de l'OTAN est un succès puisque le Conseil de l'Atlantique Nord a décidé, le 30 septembre 2005, de prolonger son soutien à l'Union africaine jusqu'au 31 mars 2006 en continuant d'une part, le transport des troupes et d'autre part, la formation des officiers ${ }^{139}$. Cette décision fait suite à une demande de l'UA qui souhaitait davantage d'appui de la part de l'OTAN, marquant ainsi pour l'Organisation sa capacité à apporter une présence significative au Darfour afin de contribuer à l'arrêt de la violence.

Le 2 juin $2006^{140}$, le président de la Commission de l'Union africaine, M. Kounaré, a demandé officiellement à l'OTAN un soutien supplémentaire pour la constitution de capacités et l'aide à la formation des troupes. Le 7 juin, le Conseil de l'Atlantique Nord a décidé de prolonger jusque fin 2006 l'assistance en matière de transport aérien fournie par l'OTAN à l'Union africaine. Le 14

138. "NATO diversifies aid to African Union" (11 mars 2007), en ligne : OTAN <http://www.nato.int/shape/news/2005/08/050811a.htm>.

139. "NATO continues to offer logistical Support to African Union" (11 mars 2007), en ligne : OTAN

<http://www.shape.nato.int/shape/news/2005/10/051006c.htm>.

140. "L'assistance de l'OTAN à l'Union africaine pour le Darfour; Quelles sont les origines de cette assistance?» (11 mars 2007), en ligne: OTAN <http://www.nato.int/issues/ darfur/evolution-f.html>. 
décembre 2006, l'OTAN a décidé de prolonger de six mois sa mission de soutien et, le 15 janvier dernier, l'Alliance a accepté d'assurer la formation du personnel au QG de la Mission de l'UA à Khartoum, en plus de la formation dispensée aux QG d'El Fasher et d'Addis Abeba141.

Au final, l'action de l'OTAN qui a été plus limitée que celle de l'UE'142 a pourtant été plus visible et il apparaît que bon nombre d'officiers ignorent le rôle de l'UE. Il n'est donc pas étonnant que le Commissaire de l'Union africaine pour la paix et la sécurité, $M$. Saïd Djinnit, se soit rendu au siège de l'OTAN le 2 mars dernier, afin d'aborder le problème de la détérioration de la situation dans la région au niveau de la sécurité, et plus largement, les besoins à long terme de l'Union africaine en matière de développement des capacités et de formation. Il a, à cette occasion, demandé à l'OTAN d'étudier la possibilité d'étendre la coopération à d'autres domaines ${ }^{143}$.

Cette arrivée de l'OTAN en Afrique n'est qu'une première étape. En 2006, des manœuvres de la Force de réaction rapide, prévues initialement en Mauritanie, ont déjà eu lieu dans les îles africaines du Cap-Vert. C'était la première fois qu'un exercice d'une telle ampleur était organisé en Afrique. L'opération baptisée Livex 06 a impliqué plusieurs milliers de soldats et a testé toutes les composantes (terrestre, maritime et aéroportée) de la NRF144. La présence de l'OTAN en Afrique symbolise la conséquence de sa transformation et la fin d'une époque où les anciennes puissances

141. Ibid.

142. L'UE est intervenue, tant sur le plan militaire qu'humanitaire, en tant que principal bailleur de fonds mais n'a pas su s'imposer comme partenaire privilégié de l'UA. Marie Molinié, "La coopération entre l'UE et l'UA au Darfour: Un bilan contrasté" (11 mars 2007), en ligne : Le Taurillon <http://www.taurillon.org/La-cooperation-entre-1-UE-et-1-UA> [La coopération entre l'UE et l'UA au Darfour ].

143. "L'Union africaine souhaite établir une coopération à long terme avec l'OTAN" (11 mars 2007), en ligne : OTAN <http://www.nato.int/docu/ update/2007/03-march/f0302a.html>.

144. "L'Otan va tester pour la première fois sa force de réaction en Afrique" (11 mars 2007), en ligne: AMI <http://www.ami.mr/fr/Archives2005/ avril/12/10.htm>. 
coloniales y jouaient seules un rôle ${ }^{145}$. L'appui de l'OTAN à la mission de l'Union africaine a facilité "la création de liens entre les principales organisations régionales»" ${ }^{146}$ sur le continent africain et devrait marquer le commencement d'une implication de plus en plus grande de l'Organisation dans cette région, dans le cadre d'opérations de maintien de la paix mandatées par les Nations Unies. Cette dernière condition est fondamentale parce qu'elle permet, d'une part, l'établissement d'un consensus au sein de l'Alliance en matière d'intervention et, d'autre part, une certaine légitimation internationale quant au rôle mondial de l'OTAN que l'Alliance entend développer en agissant en dehors de sa zone d'influence originelle.

En conclusion, malgré l'évolution de l'Alliance consécutive aux changements géopolitiques, les membres mêmes de l'OTAN ne sont pas unanimes sur la fonction de l'Organisation. Pour les États-Unis, l'actualisation et la diversification des tâches montrent que l'OTAN a toujours un rôle et doit rester une alternative pour assurer la défense territoriale des Alliés et surtout pour entreprendre, au besoin, dans le cadre de l'OTAN, des missions non article 5 de gestion de crises. Pour les États européens, membres originaires, l'OTAN a longtemps symbolisé la protection américaine face à la menace soviétique. Elle reste pour certains le fondement de la défense collective alors que, pour d'autres, dont la France et l'Allemagne, il est nécessaire d'édifier une défense européenne autonome. Pour les nouveaux membres européens, elle représente une assurance protectrice vis-à-vis de la Russie en même temps qu'un gage de progrès dans les réformes de leurs armées par le biais du processus d'intégration du PPP puis du

145. Antoine Sadoux, "La PESD : un moyen d'assurer la position de la France en Afrique ?" (2005) 10 Défense nationale et sécurité collective 67 aux pp. 69-77.

146. Déclaration du général James Jones, commandant en chef des forces américaines en Europe et commandant suprême des forces alliées en Europe (SACEUR), à l'occasion d'une déposition devant la commission sénatoriale des relations étrangères, Washington, DC, 28 septembre 2005. "U.S. Military Missions in Africa", <http://www.senate.gov/ foreign/testimony/2005/Jones Testimony050928.pdf>. 
Plan d'action pour l'adhésion (MAP) ${ }^{147}$. Finalement, pour tous, l'Alliance est le cadre de coopération politico-militaire les liant de manière permanente entre eux et surtout aux États-Unis. L'OTAN doit perdurer au moins pour demeurer une enceinte dans laquelle Américains et Européens peuvent discuter de leurs intérêts stratégiques.

Sans contredit, l'une des plus grandes évolutions qu'a récemment connue l'OTAN est la disparition des références aux zones article 5 ou non-article 5. En effet, depuis Prague en 2002, le facteur géographique a perdu de sa pertinence. L'OTAN intervient désormais là où son intérêt la guide, c'est-à-dire vers de nouveaux théâtres géographiques comme en Afrique, dans de nouvelles missions comme c'est le cas de la lutte contre le terrorisme ou de l'aide humanitaire et avec de nouveaux instruments comme la NRF. Elle évolue donc vers une véritable organisation de sécurité, au sens large.

Cette nouvelle vision élargie de la mission de l'OTAN s'accompagne de l'accroissement des capacités militaires de l'Organisation et de l'adaptation des forces armées qui deviennent plus souples et plus aisément déployables dans le cadre de la NRF. Cependant, malgré la mise en place de ces nouveaux moyens créés pour s'adapter au nouvel environnement stratégique, les membres alliés ont de plus en plus de mal à se mettre d'accord sur l'usage de cette force. On peut donc imaginer qu'à l'avenir, les missions de l'OTAN pourraient prendre trois formes. Tout d'abord, l'OTAN pourra conduire des opérations de maintien de la paix et d'autres opérations d'après conflit sous son propre drapeau, comme ce fut le cas au Kosovo. Ensuite, l'OTAN pourra servir de cadre pour aider les coalitions de pays déterminés à agir, comme en Afghanistan. Enfin, l'Organisation pourra servir de sous-

147. Le Plan d'action pour l'adhésion (MAP) doit aider les pays qui souhaitent entrer dans l'Alliance en leur donnant conseils, aide et soutien pratique concernant tous les aspects de l'adhésion à l'OTAN. "Le Plan d'action pour l'adhésion à l'OTAN» (11 mars 2007), en ligne : U.S. Department of State <http://usinfo.state.gov>. 
contractant pour les opérations de maintien de la paix et de sanction de l'ONU, comme au Darfour.

La transformation de l'OTAN a aussi été abordée lors du sommet de Riga des 28 et 29 novembre 2006 ${ }^{148}$. Toutefois, plusieurs points, pourtant évoqués dans l'ordre du jour ${ }^{149}$, n'ont pas été discutés. D'une part, l'Alliance ne s'est pas prononcée sur le financement commun des opérations qui jusque-là était supporté seulement par les États qui étaient engagés. Sur ce point, les chefs d'État et de gouvernement ont seulement encouragé les États à augmenter les "dépenses de défense en termes réels» ${ }^{150}$. D'autre part, le sommet de Riga devait aborder la question d'un nouveau type de partenariat mondial renforcé avec des pays qui "défendent [ses] valeurs et préoccupations en matière de sécurité» ${ }^{151}$ comme l'Australie, la Nouvelle-Zélande, la Corée du Sud et le Japon. Or, ces pays n'ont même pas été mentionnés lors du sommet. Finalement, la discussion sur l'élargissement futur de l'OTAN, qui devait être l'un des thèmes principaux du sommet, a perdu beaucoup de son importance. Pour l'Albanie, la Croatie et l'ex-République yougoslave de Macédoine, des négociations d'accession pourront être ouvertes en 2008152. Pour la Géorgie et l'Ukraine, seule la possibilité d'ouvrir un dialogue a été indiquée 153. L'Alliance a invité la Bosnie-Herzégovine, le Monténégro et la Serbie à adhérer au Partenariat pour la paix et au Conseil de partenariat euro-atlantique, ce qui est un premier pas vers l'intégration et constitue un revirement de position, puisque l'Alliance avait toujours posé la condition de l'arrestation de Mladic et Karadzic comme préalable à la poursuite des relations avec la Serbie.

\footnotetext{
148. "Déclaration du Sommet de Riga" (11 mars 2007), en ligne : OTAN <http: //www.nato.int/docu/pr/2006/p06-150f.htm>.

149. Jaap de Hoop Scheffer, "Quelques réflexions à propos du Sommet de Riga" (11 mars 2007), en ligne : OTAN <http://www.nato.int/docu/ review/2006/issue4/french/art1.html>.

150. Déclaration du Sommet de Riga, supra note 148 au point 26.

151. La coopération entre l'UE et l'UA au Darfour, supra note 142.

152. Déclaration du Sommet de Riga, supra note 148 au point 30.

153. Ibid. point 37.
} 
L'un des thèmes majeurs de ce sommet a été l'Afghanistan. Toutefois, même si ce sujet occupe les points 4 à 8 de la Déclaration finale du sommet, aucune décision, quant à l'augmentation des moyens déployés, n'a été prise ${ }^{154}$. Mais, les faibles avancées sur le dossier afghan mettent surtout en lumière une vision stratégique différente entre les États-Unis et les Européens. Les premiers souhaitant élargir l'OTAN afin qu'elle devienne davantage une alliance mondiale155 qu'une alliance transatlantique, alors que les seconds souhaitent s'exempter de la tutelle américaine et veulent éviter que l'Alliance ne soit formée de troupes à la disposition des États-Unis.

D’autres points ont été abordés. D’une part, les chefs d'État et de gouvernement ont souligné le rôle de catalyseur de la NRF "pour la transformation et l'interopérabilité"156. Ils ont réaffirmé que la NRF avait atteint sa "capacité opérationnelle finale»157.

154. La seule décision nouvelle est à l'initiative et porte sur la création d'un groupe de contact destiné à favoriser l'émergence d'une solution politique globale. Ce groupe réunirait "les pays de la région, les pays contributeurs de troupes (y compris des pays non membres de l'OTAN comme la Nouvelle-Zélande) et les organisations internationales (ONU, Banque mondiale notamment)". "La France propose un groupe de contact sur l'Afghanistan" (11 mars 2007), en ligne : La représentation permanente de la France au Conseil de l'Atlantique Nord <http://www.rpfranceotan.org/article.php3?id_article=435>. Toutefois, le secrétaire général de l'OTAN, Jaap de Hoop Scheffer, a déclaré que ce projet avait peu de chances de voir le jour, précisant que "[l']on s'oriente plutôt vers l'amélioration des cénacles de discussion existants". "Chronologie FIAS : janvier 2007" (11 mars 2007), en ligne: Operations de paix<http://www.operationspaix.net/ spip.php?page $=$ chronologie\&id_mot $=85 \&$ date $=2007-01>$.

155. C'est pourquoi les États-Unis soutenaient le rapprochement avec l'Australie, le Japon, la Corée du Sud et la Nouvelle-Zélande.

156. Déclaration du Sommet de Riga, supra note 148 au point 23.

157. Ibid. De plus, afin de poursuivre l'adaptation des forces, les chefs d'État et de gouvernement ont pris une série de mesures, telles l'augmentation des moyens de transport stratégique, la transformation des forces d'opérations spéciales, l'aptitude à apporter un soutien militaire aux opérations de stabilisation et aux efforts de reconstruction dans toutes les phases d'une crise..., point 24 . 
D'autre part, la Directive politique globale158 qui donne un "cadre et des orientations politiques pour la poursuite de la transformation de l'OTAN" 159 vers les 10 à 15 prochaines années, a été adoptée bien qu'elle soit moins ambitieuse que lors de la préparation du sommet au printemps 2006. Cependant, il faut souligner la reconnaissance explicite du rôle de l'UE en matière de sécurité internationale ${ }^{160}$.

En conclusion, le sommet de Riga a fait ressortir des divergences sur la vision stratégique et le rôle de l'OTAN que l'on croyait disparues. Cependant, ces dissensions sont le signe de la vitalité de l'OTAN qui demeure "un lieu en permanente effervescence»161 et dont la "crise interne lui sert [...] à évoluer»162.

158. "Directive politique globale; Entérinée par les chefs d'État et de gouvernement de l'OTAN" (11 mars 2007), en ligne : OTAN <http://www.nato.int/docu/ basictxt/b061129f.htm>.

159. Déclaration du Sommet de Riga, supra note 148 au point 2 .

160. Ibid. au point 41.

161. Yves Boyer, "L'OTAN après le Sommet de Riga" (11 mars 2007), en ligne : Fondation pour la recherche stratégique <http://www.frstrategie.org/ barreCompetences/ secuEuropeenne/20061220.pdf>.

162. Ibid. 


\section{Allied Command Operations (ACO) Supreme Headquarters Allied Power Europe - SHAPE - Mons, BE}

1) Joint Force Command HQ Brunssum - Brunssum, NL :

$\Rightarrow \quad$ Command Component Maritime HQ Northwood, UK

$\Rightarrow \quad$ Command Component Air HQ Ramstein, GE

$\Rightarrow \quad$ Joint Headquarters Northeast - JHQ NORTHEAST - Karup, DA (deactivated)

$\Rightarrow \quad$ Command Component Land HQ Heidelberg, GE

2) Joint Force Command HQ Naples - Naples, IT

$\Rightarrow \quad$ Command Component Maritime HQ Napels, IT

$\Rightarrow \quad$ Command Component Air HQ Izmir, TU

$\Rightarrow \quad$ Command Component Land HQ Madrid, SP

$\Rightarrow$ Joint Headquarters South - JHQ SOUTH - Verona, IT (deactivated)

$\Rightarrow$ Joint Headquarters Southcentre - JHQ SOUTHCENT Larissa, GR

$\Rightarrow \quad$ Joint Headquarters Southeast - JHQ SOUTHEAST - Izmir, TU (deactivated)

3) Joint Headquarters Lisbon - Lisbon, $P O$

4) Rapidly Deployable Corps Headquarters

$\Rightarrow \quad$ Allied Command Europe Rapid Reaction Corps (ARRC) HQ Rheindalen (Germany)

$\Rightarrow \quad$ EUROCORPS HQ in Strasbourg (France)

$\Rightarrow \quad$ Rapid Deployable Italian Corps - Milan (Italy)

$\Rightarrow \quad$ Rapid Deployable Turkish Corps HQ - Istanbul (Turkey)

$\Rightarrow \quad$ Rapid Deployable German-Netherlands Corps HQ - Münster (Germany)

$\Rightarrow \quad$ Rapid Deployable Spanish Corps HQ in Valencia (Spain) 
5) Other Staffs and Commands Responsible to SACEUR

$\Rightarrow \quad$ The Reaction Forces (Air) Staff - RF(A)S - Kalkar - GE

$\Rightarrow \quad$ NATO Airborne Early Warning Force (NAEWF)

$\Rightarrow \quad$ Immediate Reaction Forces (Maritime)

$\Rightarrow \quad A C E$ Mobile Force - AMF - Heidelberg, GE

$\Rightarrow \quad$ Standing Naval Force Atlantic - STANAVORLANT

$\Rightarrow \quad$ Standing Naval Forces Mediterranean - STANAVFORMED

$\Rightarrow \quad$ Standing Naval Forces Channel - STANAVFORCHAN

Allied Command Transformation (ACT) Headquarters Supreme Allied Commander Transformation - HQ SACT Norfolk, US

1) Joint Warfare Centre - JWC - Stavanger, NO

2) SACLANT Undersea Research Centre (SACLANTCEN) - La Spezia, IT

3) NATO School (SHAPE) - Oberammergau, GE 
